\title{
A Hilbert Space Approach to Fractional Differential Equations
}

\author{
Kai Diethelm ${ }^{1}$ (D) $\cdot$ Konrad Kitzing ${ }^{2} \cdot$ Rainer Picard $^{2} \cdot$ Stefan Siegmund $^{2}$ (D) \\ Sascha Trostorff ${ }^{3}$. Marcus Waurick ${ }^{4}$
}

Received: 6 February 2020 / Revised: 25 November 2020 / Accepted: 24 December 2020 /

Published online: 2 February 2021

(c) The Author(s) 2021

\begin{abstract}
We study fractional differential equations of Riemann-Liouville and Caputo type in Hilbert spaces. Using exponentially weighted spaces of functions defined on $\mathbb{R}$, we define fractional operators by means of a functional calculus using the Fourier transform. Main tools are extrapolation- and interpolation spaces. Main results are the existence and uniqueness of solutions and the causality of solution operators for non-linear fractional differential equations.
\end{abstract}

Keywords Fractional differential equations $\cdot$ Caputo derivative $\cdot$ Riemann-Liouville derivative $\cdot$ Causality

Mathematics Subject Classification 26A33 Fractional derivatives and integrals · 45D05 Volterra integral equations

\section{Introduction}

The goal of this paper is to develop a unified framework for discussing fractional differential operators and the associated differential equation in spaces of functions mapping to general Hilbert spaces $H$.

Specifically, traditional fractional calculus (in the case where $H=\mathbb{R}^{d}$ with some $d \in \mathbb{N}$ ) usually deals with functions defined on intervals of the form $[a, b]$ or $[a, \infty$ [ with some finite $a \in \mathbb{R}$ and then discusses initial value problems with initial conditions being given at the point $a$ (often chosen as $a=0)[3,12]$.

Stefan Siegmund

stefan.siegmund@tu-dresden.de

1 Fakultät Angewandte Natur- und Geisteswissenschaften, Hochschule für angewandte Wissenschaften Würzburg-Schweinfurt, Schweinfurt, Germany

2 Institut für Analysis, Fakultät Mathematik, TU Dresden, Dresden, Germany

3 Mathematisches Seminar, Christian-Albrechts-Universität zu Kiel, Kiel, Germany

4 Institut für Angewandte Analysis, Fakultät für Mathematik und Informatik, TU Bergakademie Freiberg, Freiberg, Germany 
On the other hand, the techniques from functional analysis and functional/operational calculus that we will use are most naturally specified in the context of functions defined on the entire real line. This is also the setting preferred by certain engineers and scientists that apply fractional derivatives in areas like signal processing and systems theory where causality is a desired property, cf., e.g., [7].

The concept of a fractional derivative $\left.\left.\partial_{0}^{\alpha}, \alpha \in\right] 0,1\right]$, which we utilize, will be based on inverting a suitable continuous extension of the Riemann-Liouville fractional integral of continuous functions $f \in C_{\mathrm{c}}(\mathbb{R})$ with compact support given by

$$
t \mapsto \frac{1}{\sqrt{2 \pi}} \int_{-\infty}^{t} \frac{1}{\Gamma(\alpha)}(t-s)^{\alpha-1} f(s) \mathrm{d} s
$$

as an apparently natural interpolation suggested by the iterated kernel formula for repeated integration. The choice of the lower limit as $-\infty$ is determined by our wish to study dynamical processes, for which causality ${ }^{1}$ should play an important role.

It is a pleasant fact that the classical definition of $\partial_{0}^{\alpha}$ in the sense of [2] coincides with the other natural choice of $\partial_{0}^{\alpha}$ as a function of $\partial_{0}$ in the sense of a spectral functional calculus of a realization of $\partial_{0}$ as a normal operator in a suitable Hilbert space setting. This is specified below.

The Hilbert space framework is based on observations in [9] and has already been exploited for linear fractional partial differential equations in [10]. In this paper, however, we study fractional differentiation and different notions of nonlinear fractional differential equations, using extrapolated fractional Sobolev spaces.

The approach taken in this paper contrasts with other approaches in fractional calculus. Indeed, in [13] a fractional derivative is defined as a derivative of a fractional integral, in [4] the fractional derivative of $\mathbb{C}$-valued functions on a bounded interval and linear fractional differential equations are also studied with a functional calculus and fractional Sobolev spaces. Here, using the above mentioned functional calculus of the derivative operator, we obtain a causal implementation of the fractional derivative. The property of causality is not shared by the fractional derivative operator constructed in [4] (cf. [4, Formula (2.3)]).

Our approach to study fractional differential equations is to identify initial value problems involving fractional derivatives as a fixed point problem in a suitable Hilbert space. More precisely, we will show that equations dealing with both standard cases of fractional derivatives, the Riemann-Liouville derivative and the Caputo derivative, can be studied as such a fixed point problem, however in different spaces. A similar idea was already used by three of the authors in [5] to study differential equations with delay effects.

The article is structured as follows. We begin to introduce the Hilbert space setting in Sect. 2 and to define the fractional derivative in terms of a functional calculus. Moreover, we provide an explicit representation formula for the fractional integral in Theorem 2.6. Section 3 is devoted to the definition and basic properties of inter- and extrapolation spaces associated with the differentiation operator introduced in Sect. 2. In contrast to the spaces used in [5] and [9] we will need a continuous scale of extrapolation spaces to deal with fractional differential equations. In Sect. 4 we provide the abstract solution theory for fractional differential

${ }^{1}$ Other frequent choices such as

$$
t \mapsto \frac{1}{\sqrt{2 \pi}} \chi_{] a, \infty[}(t) \int_{a}^{t} \frac{1}{\Gamma(\alpha)}(t-s)^{\alpha-1} f(s) \mathrm{d} s
$$

for $a \in \mathbb{R}$, would lose time-shift invariance (a suggestive choice is $a=0$ ), which we consider undesirable. For our choice of the limit case $a=-\infty$ it should be noted that the Liouville and the Caputo fractional derivative essentially coincide. 
equations posed as functional equations in the spaces introduced before. More precisely, we consider equations of the form

$$
\partial_{0}^{\alpha} u=F(u)
$$

for suitable functions $F$, which act on extrapolation spaces and satisfy a certain Lipschitzcondition and show that these equations can be solved easily using the contraction mapping theorem. Moreover, we address the issue of causality for the associated solution operator. In the concluding section we study classical initial value problems for Riemann-Liouville and Caputo fractional derivatives, as they are treated in the literature and show that both can be reformulated as equations of the form (1.1) and their well-posedness is a direct consequence of the abstract results obtained in the previous section.

\section{Fractional Derivative in a Hilbert Space Setting}

In the present section, we introduce the necessary operators to be used in the following. We will formulate all results in the vector-valued, more specifically, in the Hilbert space-valued situation.

To begin with, we introduce an $L^{2}$-variant of the exponentially weighted space of continuous functions that proved useful in the proof of the Picard-Lindelöf Theorem and is attributed to Morgenstern, [6].

We denote by $L^{p}(\mathbb{R} ; H)$ and $L_{\text {loc }}^{1}(\mathbb{R} ; H)$ the space of $p$-Bochner integrable functions and the space of locally Bochner integrable functions on a Hilbert space $H$, respectively.

Definition Let $H$ be a Hilbert space, $\varrho \in \mathbb{R}$ and $p \in[1, \infty]$. For $f \in L_{\text {loc }}^{1}(\mathbb{R} ; H)$ we denote $\mathrm{e}^{-\varrho m} f:=\left(\mathbb{R} \ni t \mapsto \mathrm{e}^{-\varrho t} f(t)\right)$. We define the normed spaces

$$
L_{\varrho}^{p}(\mathbb{R} ; H):=\left\{f \in L_{\mathrm{loc}}^{1}(\mathbb{R} ; H) ; \mathrm{e}^{-\varrho m} f \in L^{p}(\mathbb{R} ; H)\right\},
$$

with norm

$$
\begin{array}{ll}
\|f\|_{L_{\varrho}^{p}(\mathbb{R} ; H)}:=\left\|\mathrm{e}^{-\varrho m} f\right\|_{L^{p}(\mathbb{R} ; H)}=\left(\int_{\mathbb{R}}\|f(t)\|_{H}^{p} \mathrm{e}^{-p \varrho t} \mathrm{~d} t\right)^{1 / p} & (p<\infty), \\
\|f\|_{L_{\varrho}^{p}(\mathbb{R} ; H)}:=\left\|\mathrm{e}^{-\varrho m} f\right\|_{L^{p}(\mathbb{R} ; H)}=\operatorname{ess} \sup \left\|\mathrm{e}^{-\varrho m} f\right\|_{H} & (p=\infty) .
\end{array}
$$

Remark 2.1 The operator $\mathrm{e}^{-\varrho m}: L_{\varrho}^{p}(\mathbb{R} ; H) \rightarrow L^{p}(\mathbb{R} ; H), f \mapsto \mathrm{e}^{-\varrho m} f$ is an isometric isomorphism from $L_{\varrho}^{p}(\mathbb{R} ; H)$ to $L^{p}(\mathbb{R} ; H)$. Moreover $L_{\varrho}^{2}(\mathbb{R} ; H)$ is a Hilbert space with scalar product

$$
(f, g) \mapsto\langle f, g\rangle_{L_{\varrho}^{2}(\mathbb{R} ; H)}=\int_{\mathbb{R}}\langle f(t), g(t)\rangle_{H} \mathrm{e}^{-2 \varrho t} \mathrm{~d} t
$$

Next, we introduce the time derivative.

Definition Let $H$ be a Hilbert space.

1. Let $f, g \in L_{\mathrm{loc}}^{1}(\mathbb{R} ; H)$. We say that $f^{\prime}=g$, if for all $\phi \in C_{c}^{\infty}(\mathbb{R})$

$$
-\int_{\mathbb{R}} f \phi^{\prime}=\int_{\mathbb{R}} g \phi .
$$


2. Let $\varrho \in \mathbb{R}$. We define

$$
\begin{aligned}
\partial_{0, \varrho}: H_{\varrho}^{1}(\mathbb{R} ; H) \subseteq L_{\varrho}^{2}(\mathbb{R} ; H) & \rightarrow L_{\varrho}^{2}(\mathbb{R} ; H) \\
f & \mapsto f^{\prime},
\end{aligned}
$$

where $H_{\varrho}^{1}(\mathbb{R} ; H):=\left\{f \in L_{\varrho}^{2}(\mathbb{R} ; H) ; f^{\prime} \in L_{\varrho}^{2}(\mathbb{R} ; H)\right\}$.

The index 0 in $\partial_{0, \varrho}$ shall indicate that the derivative is with respect to time. We will introduce the fractional derivatives and fractional integrals by means of a functional calculus for $\partial_{0, \varrho}$. For this, we introduce the Fourier-Laplace transform.

Definition Let $H$ be a complex Hilbert space and $\varrho \in \mathbb{R}$. We recall that the Fourier transform can be established as a unitary mapping $\mathcal{F}: L^{2}(\mathbb{R} ; H) \rightarrow L^{2}(\mathbb{R} ; H)$, which for $g \in$ $L^{1}(\mathbb{R} ; H) \cap L^{2}(\mathbb{R} ; H)$ is given by

$$
\mathcal{F} g(\xi)=\frac{1}{\sqrt{2 \pi}} \int_{\mathbb{R}} g(t) e^{-\mathrm{i} \xi t} \mathrm{~d} t, \quad \xi \in \mathbb{R} .
$$

We define the Fourier-Laplace transform on $L_{\varrho}^{2}(\mathbb{R} ; H)$ as the unitary mapping $\mathcal{L}_{\varrho}:=\mathcal{F} \mathrm{e}^{-\varrho m}$ : $L_{\varrho}^{2}(\mathbb{R} ; H) \rightarrow L^{2}(\mathbb{R} ; H)$

From now on, $H$ denotes a complex Hilbert space. With the latter notion at hand, we provide the spectral representation of $\partial_{0, \varrho}$ as the multiplication-by-argument operator

$$
\begin{aligned}
& \operatorname{dom}(m):=\left\{f \in L^{2}(\mathbb{R} ; H) ;(\mathbb{R} \ni \xi \mapsto \xi f(\xi)) \in L^{2}(\mathbb{R} ; H)\right\}, \\
& m: L^{2}(\mathbb{R} ; H) \supseteq \operatorname{dom}(m) \rightarrow L^{2}(\mathbb{R} ; H), \quad f \mapsto(\mathbb{R} \ni \xi \mapsto \xi f(\xi)) .
\end{aligned}
$$

Theorem 2.2 Let $\varrho \in \mathbb{R}$. Then

1. $\partial_{0,0}=\mathcal{F}^{*} \mathrm{i} m \mathcal{F}$,

2. $\left(\mathrm{e}^{-\varrho m}\right)^{*} \partial_{0,0} \mathrm{e}^{-\varrho m}=\partial_{0, \varrho}-\varrho$,

3. $\partial_{0, \varrho}=\mathcal{L}_{\varrho}^{*}(\mathrm{i} m+\varrho) \mathcal{L}_{\varrho}$.

Proof For the proof of (a) we refer to [1, Vol 1, p.161-163]. Part (b) can easily obtained by the product rule and (c) follows from (a) and (b).

Theorem 2.2 tells us that $\partial_{0, \varrho}$ is unitarily equivalent to a multiplication operator with spectrum equal to $i \mathbb{R}+\varrho=\{z \in \mathbb{C} ; \operatorname{Re} z=\varrho\}$. In particular, we are now in the position to define functions of $\partial_{0, \varrho}$.

Definition Let $\varrho \in \mathbb{R}$ and $F: \operatorname{dom}(F) \subseteq\{$ it $+\varrho ; t \in \mathbb{R}\} \rightarrow \mathbb{C}$ be measurable such that $\{t \in \mathbb{R} ; \mathrm{i} t+\varrho \notin \operatorname{dom}(F)\}$ has Lebesgue measure zero. We define

$$
F\left(\partial_{0, \varrho}\right):=\mathcal{L}_{\varrho}^{*} F(\mathrm{i} m+\varrho) \mathcal{L}_{\varrho},
$$

where

$$
F(\mathrm{i} m+\varrho) f:=(\mathbb{R} \ni \xi \mapsto F(\mathrm{i} \xi+\varrho) f(\xi))
$$

in case $f \in L^{2}(\mathbb{R} ; H)$ is such that $(\xi \mapsto F(\mathrm{i} \xi+\varrho) f(\xi)) \in L^{2}(\mathbb{R} ; H)$.

We record an elementary fact on multiplication operators. 


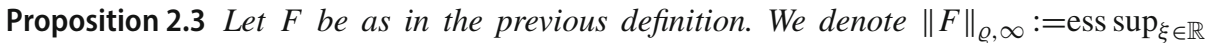
$|F(\mathrm{i} \xi+\varrho)| \in[0, \infty]$. The operator $F\left(\partial_{0, \varrho}\right)$ is bounded, if and only if $\|F\|_{\varrho, \infty}<\infty$. If $F\left(\partial_{0, \varrho}\right)$ is bounded, then $\left\|F\left(\partial_{0, \varrho}\right)\right\|=\|F\|_{\varrho, \infty}$.

Proof It is well-known that multiplication operators are bounded if and only if they stem from a bounded function. Thus, the assertion follows from the unitarity of $\mathcal{L}_{\varrho}$.

One important class of operators that can be rooted to be of the form just introduced are fractional derivatives and fractional integrals:

Example 2.4 Let $\alpha>0$ and $\varrho \in \mathbb{R}$. Then the fractional derivative of order $\alpha$ is given by

$$
\partial_{0, \varrho}^{\alpha}=\mathcal{L}_{\varrho}^{*}(\mathrm{i} m+\varrho)^{\alpha} \mathcal{L}_{\varrho}
$$

and the fractional integral of order $\alpha$ is given by

$$
\partial_{0, \varrho}^{-\alpha}=\mathcal{L}_{\varrho}^{*}\left(\frac{1}{\mathrm{i} m+\varrho}\right)^{\alpha} \mathcal{L}_{\varrho} .
$$

Note that both expressions are well-defined in the sense of functions of $\partial_{0, \varrho}$ defined above and that $\partial_{0, \varrho}^{-\alpha}$ is bounded if and only if $\varrho \neq 0$. Moreover, $\left(\partial_{0, \varrho}^{\alpha}\right)^{-1}=\partial_{0, \varrho}^{-\alpha}$. We set $\partial_{0, \varrho}^{0}$ as the identity operator on $L_{\varrho}^{2}(\mathbb{R} ; H)$.

In order to provide the connections to the more commonly known integral representation formulas for the fractional integrals, we recall the multiplication theorem, that is,

$$
\sqrt{2 \pi} \mathcal{F} f \cdot \mathcal{F} g=\mathcal{F}(f * g),
$$

for $f \in L^{1}(\mathbb{R})$ and $g \in L^{2}(\mathbb{R} ; H)$.

We recall the cut-off function

$$
\chi_{\mathbb{R}_{>0}}(t):= \begin{cases}1, & t>0, \\ 0, & t \leq 0 .\end{cases}
$$

Lemma 2.5 For all $\varrho, \alpha>0$, and $\xi \in \mathbb{R}$, we have

$$
\sqrt{2 \pi} \mathcal{L}_{\varrho}\left(t \mapsto \frac{1}{\Gamma(\alpha)} t^{\alpha-1} \chi_{\mathbb{R}_{>0}}(t)\right)(\xi)=\left(\frac{1}{i \xi+\varrho}\right)^{\alpha} .
$$

Proof We start by defining the function

$$
f(\xi):=\int_{0}^{\infty} \mathrm{e}^{-(\mathrm{i} \xi+\varrho) s} s^{\alpha-1} \mathrm{~d} s
$$

for $\xi \in \mathbb{R}$. Then we have

$$
\begin{aligned}
f^{\prime}(\xi) & =\int_{0}^{\infty}-\mathrm{ie}^{-(\mathrm{i} \xi+\varrho) s} s^{\alpha} \mathrm{d} s \\
& =-\mathrm{i} \frac{\alpha}{\mathrm{i} \xi+\varrho} f(\xi),
\end{aligned}
$$

where we have used integration by parts. By separation of variables, it follows that

$$
f(\xi)=f(0) \frac{\varrho^{\alpha}}{(\mathrm{i} \xi+\varrho)^{\alpha}}
$$


for $\xi \in \mathbb{R}$. Now, since

$$
f(0)=\int_{0}^{\infty} \mathrm{e}^{-\varrho s} s^{\alpha-1} \mathrm{~d} s=\frac{1}{\varrho^{\alpha}} \Gamma(\alpha),
$$

we infer

$$
f(\xi)=\Gamma(\alpha) \frac{1}{(\mathrm{i} \xi+\varrho)^{\alpha}} .
$$

Since the left hand side of (2.1) equals $\frac{1}{\Gamma(\alpha)} f(\xi)$, the assertion follows.

Next, we draw the connection from our fractional integral to the one used in the literature.

Theorem 2.6 For all $\varrho, \alpha>0, f \in L_{\varrho}^{2}(\mathbb{R} ; H)$ and $t \in \mathbb{R}$, we have

$$
\partial_{0, \varrho}^{-\alpha} f(t)=\int_{-\infty}^{t} \frac{1}{\Gamma(\alpha)}(t-s)^{\alpha-1} f(s) \mathrm{d} s .
$$

Proof. We set $g:=\left(\mathbb{R} \ni t \mapsto \frac{1}{\Gamma(\alpha)} t^{\alpha-1} \chi_{\mathbb{R}_{>0}}(t)\right)$. Then $g \in L_{\varrho}^{1}(\mathbb{R})$. For $f \in L_{\varrho}^{2}(\mathbb{R} ; H)$ we have by Young's convolution inequality

$$
\left(e^{-\varrho m} g\right) *\left(e^{-\varrho m} f\right)=e^{-\varrho m}(g * f) \in L^{2}(\mathbb{R} ; H) .
$$

Using the convolution property of the Fourier transform we obtain

$$
\sqrt{2 \pi} \mathcal{L}_{\varrho} g \cdot \mathcal{L}_{\varrho} f=\mathcal{L}_{\varrho}(g * f) .
$$

Using Lemma 2.5 we compute

$$
\begin{aligned}
\partial_{0, \varrho}^{-\alpha} f & =\mathcal{L}_{\varrho}^{*}\left(\frac{1}{\mathrm{i} m+\varrho}\right)^{\alpha} \mathcal{L}_{\varrho} f \\
& =\mathcal{L}_{\varrho}^{*}\left(\sqrt{2 \pi} \mathcal{L}_{\varrho} g \cdot \mathcal{L}_{\varrho} f\right) \\
& =\mathcal{L}_{\varrho}^{*} \mathcal{L}_{\varrho}(g * f) \\
& =\int_{-\infty}^{(\cdot)} \frac{1}{\Gamma(\alpha)}((\cdot)-s)^{\alpha-1} f(s) \mathrm{d} s .
\end{aligned}
$$

Corollary 2.7 Let $\varrho, \alpha>0$. Then for all $t \in \mathbb{R}$, we have for $h \in H$

$$
\left(\partial_{0, \varrho}^{-\alpha} \chi_{\mathbb{R}_{>0}} h\right)(t)= \begin{cases}\frac{1}{\Gamma(\alpha+1)} t^{\alpha} h, & t>0, \\ 0, & t \leq 0 .\end{cases}
$$

Proof We use Theorem 2.6 and obtain for $t \leq 0$

$$
\left(\partial_{0, \varrho}^{-\alpha} \chi_{\mathbb{R}_{>0}} h\right)(t)=\int_{-\infty}^{t} \frac{1}{\Gamma(\alpha)}(t-s)^{\alpha-1} \chi_{\mathbb{R}_{>0}}(s) h \mathrm{~d} s=0,
$$

since the integrand has positive support. For $t>0$, we obtain

$$
\begin{aligned}
\left(\partial_{0, \varrho}^{-\alpha} \chi_{\mathbb{R}_{>0}} h\right)(t) & =\int_{0}^{t} \frac{1}{\Gamma(\alpha)}(t-s)^{\alpha-1} \mathrm{~d} s h \\
& =\int_{0}^{t} \frac{1}{\Gamma(\alpha)} s^{\alpha-1} \mathrm{~d} s h=\frac{1}{\Gamma(\alpha)} \frac{1}{\alpha} t^{\alpha} h .
\end{aligned}
$$


Remark 2.8 It seems to be hard to determine analogous formulas for the case $\varrho<0$, although the operator $\partial_{0, \varrho}^{-\alpha}$ for $\varrho<0, \alpha>0$ is bounded. The reason for this is that the corresponding multiplier $(\mathrm{i} m+\varrho)^{-\alpha}$ is not defined in 0 and has a jump there. In particular, it cannot be extended to an analytic function on some right half plane of $\mathbb{C}$. This, however, corresponds to the causality or anticausality of the operator $\partial_{0, \varrho}^{-\alpha}$ by a Paley-Wiener result ([8] or [11, 19.2 Theorem]) and hence, we cannot expect to get a convolution formula as in the case $\varrho>0$.

\section{Extra- and Interpolation Spaces}

We begin to define extra- and interpolation spaces associated with the fractional derivative $\partial_{0, \varrho}^{\alpha}$ for $\varrho \neq 0, \alpha \in \mathbb{R}$. Since by definition

$$
\partial_{0, \varrho}^{\alpha}=\mathcal{L}_{\varrho}^{*}(\mathrm{i} m+\varrho)^{\alpha} \mathcal{L}_{\varrho},
$$

we will define the extra- and interpolation spaces in terms of the multiplication operators $(\mathrm{i} m+\varrho)^{\alpha}$ on $L^{2}(\mathbb{R} ; H)$.

Definition Let $\varrho \neq 0$. For each $\alpha \in \mathbb{R}$ we define the space

$$
H^{\alpha}(\mathrm{i} m+\varrho):=\left\{f \in L_{\mathrm{loc}}^{1}(\mathbb{R} ; H) ; \int_{\mathbb{R}}\left\|(\mathrm{i} t+\varrho)^{\alpha} f(t)\right\|_{H}^{2} \mathrm{~d} t<\infty\right\}
$$

and equip it with the natural inner product

$$
\langle f, g\rangle_{H^{\alpha}(\mathrm{i} m+\varrho)}:=\int_{\mathbb{R}}\left\langle(\mathrm{i} t+\varrho)^{\alpha} f(t),(\mathrm{i} t+\varrho)^{\alpha} g(t)\right\rangle_{H} \mathrm{~d} t
$$

for each $f, g \in H^{\alpha}(\mathrm{i} m+\varrho)$.

We shall use $X \hookrightarrow Y$ to denote the mapping $X \ni x \mapsto x \in Y$, if $X \subseteq Y$ (under a canonical identification, which will always be obvious from the context).

Lemma 3.1 For $\varrho \neq 0$ and $\alpha \in \mathbb{R}$ the space $H^{\alpha}(\mathrm{i} m+\varrho)$ is a Hilbert space. Moreover, for $\beta>\alpha$ we have

$$
j_{\beta \rightarrow \alpha}: H^{\beta}(\mathrm{i} m+\varrho) \hookrightarrow H^{\alpha}(\mathrm{i} m+\varrho)
$$

where the embedding is dense and continuous with $\left\|j_{\beta \rightarrow \alpha}\right\| \leq|\varrho|^{\alpha-\beta}$.

Proof Note that $H^{\alpha}(\mathrm{i} m+\varrho)=L^{2}(\mu ; H)$, where $\mu$ is the Lebesgue measure on $\mathbb{R}$ weighted with the function $t \mapsto|\mathrm{i} t+\varrho|^{2 \alpha}$. Thus, $H^{\alpha}(\mathrm{i} m+\varrho)$ is a Hilbert space by the Fischer-Riesz theorem. Let now $\beta>\alpha$ and $f \in H^{\beta}(\mathrm{i} m+\varrho)$. Then

$$
\int_{\mathbb{R}}\left\|(\mathrm{i} t+\varrho)^{\alpha} f(t)\right\|_{H}^{2} \mathrm{~d} t=\int_{\mathbb{R}}\left(t^{2}+\varrho^{2}\right)^{\alpha-\beta}\left\|(\mathrm{i} t+\varrho)^{\beta} f(t)\right\|_{H}^{2} \mathrm{~d} t \leq\left(\varrho^{2}\right)^{\alpha-\beta}\|f\|_{H^{\beta}(\mathrm{i} m+\varrho)}^{2},
$$

which proves the continuity of the embedding $j_{\beta \rightarrow \alpha}$ and the asserted norm estimate. The density follows, since $C_{c}^{\infty}(\mathbb{R} ; H)$ lies dense in $H^{\gamma}(\mathrm{i} m+\varrho)$ for each $\gamma \in \mathbb{R}$. 
Definition Let $\varrho \neq 0$ and $\alpha \in \mathbb{R}$. We consider the space

$$
W_{\varrho}^{\alpha}(\mathbb{R} ; H):=\left\{u \in L_{\varrho}^{2}(\mathbb{R} ; H) ; \mathcal{L}_{\varrho} u \in H^{\alpha}(\mathrm{i} m+\varrho)\right\}
$$

equipped with the inner product

$$
\langle u, v\rangle_{\varrho, \alpha}:=\left\langle\mathcal{L}_{\varrho} u, \mathcal{L}_{\varrho} v\right\rangle_{H^{\alpha}(\mathrm{i} m+\varrho)}
$$

and set $H_{\varrho}^{\alpha}(\mathbb{R} ; H)$ as its completion with respect to the norm induced by $\langle\cdot, \cdot\rangle_{\varrho, \alpha}$.

Lemma 3.2 Let $\varrho \neq 0$.

(a) For $\alpha \geq 0$ we have that $H_{\varrho}^{\alpha}(\mathbb{R} ; H)=W_{\varrho}^{\alpha}(\mathbb{R} ; H)=\operatorname{dom}\left(\partial_{0, \varrho}^{\alpha}\right)$.

(b) The operator

$$
\mathcal{L}_{\varrho}: W_{\varrho}^{\alpha}(\mathbb{R} ; H) \subseteq H_{\varrho}^{\alpha}(\mathbb{R} ; H) \rightarrow H^{\alpha}(\mathrm{i} m+\varrho)
$$

has a unique unitary extension, which will again be denoted by $\mathcal{L}_{\varrho}$.

(c) For $\alpha, \beta \in \mathbb{R}$ with $\beta>\alpha$ we have that

$$
\iota_{\beta \rightarrow \alpha}: H_{\varrho}^{\beta}(\mathbb{R} ; H) \hookrightarrow H_{\varrho}^{\alpha}(\mathbb{R} ; H)
$$

is continuous and dense with $\left\|\iota_{\beta \rightarrow \alpha}\right\| \leq|\varrho|^{\alpha-\beta}$.

(d) For each $\beta>0$ and $\alpha \in \mathbb{R}$ the operator

$$
\partial_{0, \varrho}^{\beta}: H_{\varrho}^{\beta+|\alpha|}(\mathbb{R} ; H) \subseteq H_{\varrho}^{\alpha}(\mathbb{R} ; H) \rightarrow H_{\varrho}^{\alpha-\beta}(\mathbb{R} ; H)
$$

has a unique unitary extension, which will again be denoted by $\partial_{0, \varrho}^{\beta}$.

Proof. (a) Let $\alpha \geq 0$. For $u \in H^{\alpha}(\mathrm{i} m+\varrho)$, i.e. $u \in L_{\mathrm{loc}}^{1}(\mathbb{R} ; H)$ and $(\mathrm{i} m+\varrho)^{\alpha} u \in L^{2}(\mathbb{R} ; H)$, we infer that $u \in L^{2}(\mathbb{R} ; H)$. It follows that $u \in \operatorname{dom}\left((\mathrm{i} m+\varrho)^{\alpha}\right)$. Hence $H^{\alpha}(\mathrm{i} m+\varrho)=$ $\operatorname{dom}\left((\mathrm{i} m+\varrho)^{\alpha}\right)$. Moreover,

$$
\begin{aligned}
u \in W_{\varrho}^{\alpha}(\mathbb{R} ; H) & \Leftrightarrow u \in L_{\varrho}^{2}(\mathbb{R} ; H) \wedge \mathcal{L}_{\varrho} u \in H^{\alpha}(\mathrm{i} m+\varrho) \\
& \Leftrightarrow u \in L_{\varrho}^{2}(\mathbb{R} ; H) \wedge \mathcal{L}_{\varrho} u \in \operatorname{dom}\left((\mathrm{i} m+\varrho)^{\alpha}\right) \\
& \Leftrightarrow u \in \operatorname{dom}\left(\partial_{0, \varrho}^{\alpha}\right),
\end{aligned}
$$

by Example 2.4. Moreover, since

$$
\mathcal{L}_{\varrho}: W_{\varrho}^{\alpha}(\mathbb{R} ; H) \rightarrow H^{\alpha}(\mathrm{i} m+\varrho)
$$

is unitary, we infer that $W_{\varrho}^{\alpha}(\mathbb{R} ; H)$ is complete with respect to $\|\cdot\|_{\varrho, \alpha}=\left\|\mathcal{L}_{\varrho} \cdot\right\|_{H^{\alpha}(\mathrm{i} m+\varrho)}$, and thus $H_{\varrho}^{\alpha}(\mathbb{R} ; H)=W_{\varrho}^{\alpha}(\mathbb{R} ; H)$.

(b) Obviously,

$$
\mathcal{L}_{\varrho}: W_{\varrho}^{\alpha}(\mathbb{R} ; H) \subseteq H_{\varrho}^{\alpha}(\mathbb{R} ; H) \rightarrow H^{\alpha}(\mathrm{i} m+\varrho)
$$

is isometric by the definition of the norm on $H_{\varrho}^{\alpha}(\mathbb{R} ; H)$. Moreover, its range is dense, since $\mathcal{L}_{\varrho}^{*} \varphi \in W_{\varrho}^{\alpha}(\mathbb{R} ; H)$ for each $\varphi \in C_{c}^{\infty}(\mathbb{R} ; H)$ and thus, $C_{c}^{\infty}(\mathbb{R} ; H) \subseteq \mathcal{L}_{\varrho}\left[W_{\varrho}^{\alpha}(\mathbb{R} ; H)\right]$. Hence, the continuous extension of $\mathcal{L}_{\varrho}$ to $H_{\varrho}^{\alpha}(\mathbb{R} ; H)$ is onto and, thus, unitary.

(c) Since $\iota_{\beta \rightarrow \alpha}=\mathcal{L}_{\varrho}^{*} j_{\beta \rightarrow \alpha} \mathcal{L}_{\varrho}$, the assertion follows from Lemma 3.1. 
(d) Since,

$$
\begin{aligned}
(\mathrm{i} m+\varrho)^{\beta}: H^{\alpha}(\mathrm{i} m+\varrho) & \rightarrow H^{\alpha-\beta}(\mathrm{i} m+\varrho) \\
f & \mapsto\left(t \mapsto(\mathrm{i} t+\varrho)^{\beta} f(t)\right)
\end{aligned}
$$

is obviously unitary, we infer that for $u \in H_{\varrho}^{\beta+|\alpha|}(\mathbb{R} ; H)$

$$
\begin{aligned}
\left\|\partial_{0, \varrho}^{\beta} u\right\|_{\varrho, \alpha-\beta} & =\left\|\mathcal{L}_{\varrho} \partial_{0, \varrho}^{\beta} u\right\|_{H^{\alpha-\beta}(\mathrm{i} m+\varrho)} \\
& =\left\|(\mathrm{i} m+\varrho)^{\beta} \mathcal{L}_{\varrho} u\right\|_{H^{\alpha-\beta}(\mathrm{i} m+\varrho)} \\
& =\left\|\mathcal{L}_{\varrho} u\right\|_{H^{\alpha}(\mathrm{i} m+\varrho)} \\
& =\|u\|_{\varrho, \alpha},
\end{aligned}
$$

which shows that $\partial_{0, \varrho}^{\beta}$ is an isometry. Moreover, for $\varphi \in C_{c}^{\infty}(\mathbb{R} ; H)$, we have that $(\mathrm{i} m+\varrho)^{\gamma} \varphi \in C_{c}^{\infty}(\mathbb{R} ; H)$ for all $\gamma \in \mathbb{R}$ and thus, in particular $\mathcal{L}_{\varrho}^{*}(\mathrm{i} m+\varrho)^{-\beta} \varphi \in$ $\bigcap_{\gamma \in \mathbb{R}} H_{\varrho}^{\gamma}(\mathbb{R} ; H) \subseteq H_{\varrho}^{\beta+|\alpha|}(\mathbb{R} ; H)$. Next,

$$
\partial_{0, \varrho}^{\beta} \mathcal{L}_{\varrho}^{*}(\mathrm{i} m+\varrho)^{-\beta} \varphi=\mathcal{L}_{\varrho}^{*}(\mathrm{i} m+\varrho)^{\beta} \mathcal{L}_{\varrho} \mathcal{L}_{\varrho}^{*}(\mathrm{i} m+\varrho)^{-\beta} \varphi=\mathcal{L}_{\varrho}^{*} \varphi
$$

and thus, $\mathcal{L}_{\varrho}^{*}\left[C_{c}^{\infty}(\mathbb{R} ; H)\right] \subseteq \partial_{0, \varrho}^{\beta}\left[H_{\varrho}^{\beta+|\alpha|}(\mathbb{R} ; H)\right]$. Since $C_{c}^{\infty}(\mathbb{R} ; H)$ is dense in $H^{\alpha-\beta}(\mathrm{i} m+\varrho)$, we infer that $\mathcal{L}_{\varrho}^{*}\left[C_{c}^{\infty}(\mathbb{R} ; H)\right]$ is dense in $H_{\varrho}^{\alpha-\beta}(\mathbb{R} ; H)$ and thus, $\partial_{0, \varrho}^{\beta}$ has dense range. This completes the proof.

We conclude this section by providing an alternative perspective to elements lying in $H_{\varrho}^{\alpha}(\mathbb{R} ; H)$ for some $\alpha \in \mathbb{R}$ (with a particular focus on $\alpha<0$ ). In particular, we aim for a definition of a support for those elements which coincides with the usual support of $L^{2}$ functions in the case $\alpha \geq 0$.

Lemma 3.3 Let $\varrho \neq 0$ and $\alpha \in \mathbb{R}$. Then

$$
\begin{aligned}
\sigma_{-1}: W_{\varrho}^{\alpha}(\mathbb{R} ; H) \subseteq H_{\varrho}^{\alpha}(\mathbb{R} ; H) & \rightarrow H_{-\varrho}^{\alpha}(\mathbb{R} ; H) \\
f & \mapsto(t \mapsto f(-t))
\end{aligned}
$$

extends to a unitary operator. Moreover, for $f \in H_{\varrho}^{\alpha}(\mathbb{R} ; H)$ we have

$$
\mathcal{L}_{-\varrho} \sigma_{-1} f=\sigma_{-1} \mathcal{L}_{\varrho} f \quad \text { and } \quad \mathcal{L}_{-\varrho}^{*} \sigma_{-1} f=\sigma_{-1} \mathcal{L}_{\varrho}^{*} f .
$$

Proof For $f \in W_{\varrho}^{\alpha}(\mathbb{R} ; H)$ we have that

$$
\mathcal{L}_{-\varrho} \sigma_{-1} f=\sigma_{-1} \mathcal{L}_{\varrho} f
$$

and hence,

$$
\begin{aligned}
\int_{\mathbb{R}}\left\|(\mathrm{i} t-\varrho)^{\alpha}\left(\mathcal{L}_{-\varrho} \sigma_{-1} f\right)(t)\right\|_{H}^{2} \mathrm{~d} t & =\int_{\mathbb{R}}\left(t^{2}+\varrho^{2}\right)^{\alpha}\left\|\left(\mathcal{L}_{\varrho} f\right)(-t)\right\|_{H}^{2} \mathrm{~d} t \\
& =\int_{\mathbb{R}}\left(t^{2}+\varrho^{2}\right)^{\alpha}\left\|\left(\mathcal{L}_{\varrho} f\right)(t)\right\|_{H}^{2} \mathrm{~d} t=\|f\|_{H_{\varrho}^{\alpha}(\mathbb{R} ; H)}^{2},
\end{aligned}
$$

which proves the isometry of $\sigma_{-1}$. Moreover, $\sigma_{-1}$ has dense range, since $\sigma_{-1}\left[W_{\varrho}^{\alpha}(\mathbb{R} ; H)\right]=$ $W_{-\varrho}^{\alpha}(\mathbb{R} ; H)$. Hence, $\sigma_{-1}$ extends to a unitary operator. The equality $\mathcal{L}_{-\varrho} \sigma_{-1} f=\sigma_{-1} \mathcal{L}_{\varrho} f$ holds for $f \in H_{\varrho}^{\alpha}(\mathbb{R} ; H)$, since $W_{\varrho}^{\alpha}(\mathbb{R} ; H)$ is dense in its completion $H_{\varrho}^{\alpha}(\mathbb{R} ; H)$. 
Proposition 3.4 Let $\varrho \neq 0, \alpha \in \mathbb{R}$ and $f \in H_{\varrho}^{\alpha}(\mathbb{R} ; H)$. Then

$$
\langle f, \cdot\rangle: C_{c}^{\infty}(\mathbb{R} ; H) \rightarrow \mathbb{C}
$$

given by

$$
\langle f, \varphi\rangle:=\int_{\mathbb{R}}\left\langle\mathcal{L}_{\varrho} f(t), \mathcal{L}_{-\varrho} \varphi(t)\right\rangle_{H} \mathrm{~d} t
$$

defines a distribution. Moreover, for $f \in H_{\varrho}^{\alpha}(\mathbb{R} ; H)$ and $\varphi \in C_{c}^{\infty}(\mathbb{R} ; H)$ we have

$$
\langle f, \varphi\rangle=\left\langle f, \partial_{0, \varrho}^{-\alpha} \mathrm{e}^{2 \varrho m} \sigma_{-1} \partial_{0, \varrho}^{-\alpha} \sigma_{-1} \varphi\right\rangle_{\varrho, \alpha} .
$$

In particular, for $\alpha=0$

$$
\langle f, \varphi\rangle=\int_{\mathbb{R}}\langle f(t), \varphi(t)\rangle_{H} \mathrm{~d} t .
$$

Note that the operator $\partial_{0, \varrho}^{-\alpha} \mathrm{e}^{2 \varrho m} \sigma_{-1} \partial_{0, \varrho}^{-\alpha} \sigma_{-1}$ maps $H_{-\varrho}^{-\alpha}(\mathbb{R} ; H)$ to $H_{\varrho}^{\alpha}(\mathbb{R} ; H)$ unitarily.

Proof. Let $f \in H_{\varrho}^{\alpha}(\mathbb{R} ; H)$. We first prove that the expression $\langle f, \cdot\rangle$ is indeed a distribution. Due to Lemma 3.2(c) it suffices to prove this for $f \in H_{\varrho}^{-k}(\mathbb{R} ; H)$ for some $k \in \mathbb{N}$. Indeed, if $f \in H_{\varrho}^{-k}(\mathbb{R} ; H)$, then we know that

$$
\left(t \mapsto(\mathrm{i} t+\varrho)^{-k}\left(\mathcal{L}_{\varrho} f\right)(t)\right) \in L^{2}(\mathbb{R} ; H)
$$

and hence, for $\varphi \in C_{c}^{\infty}(\mathbb{R} ; H)$ we obtain using Hölder's inequality and the fact that $\mathcal{L}_{-\varrho} \varphi^{(k)}=(\mathrm{i} m+\varrho)^{k} \mathcal{L}_{-\varrho} \varphi$

$$
\begin{aligned}
|\langle f, \varphi\rangle| & \leq \int_{\mathbb{R}}\left|\left\langle(\mathrm{i} t+\varrho)^{-k}\left(\mathcal{L}_{\varrho} f\right)(t),(-\mathrm{i} t+\varrho)^{k}\left(\mathcal{L}_{-\varrho} \varphi\right)(t)\right\rangle_{H}\right| \mathrm{d} t \\
& \leq\left\|\mathcal{L}_{\varrho} f\right\|_{H^{-k}(\mathrm{i} m+\varrho)}\left\|\mathcal{L}_{-\varrho}\left(\varphi^{(k)}\right)\right\|_{L^{2}(\mathbb{R} ; H)} \\
& \leq\left\|\mathcal{L}_{\varrho} f\right\|_{H^{-k}(\mathrm{i} m+\varrho)}\left(\int_{\operatorname{spt} \varphi} \mathrm{e}^{2 \varrho t} \mathrm{~d} t\right)^{\frac{1}{2}}\left\|\varphi^{(k)}\right\|_{\infty},
\end{aligned}
$$

which proves that $\langle f, \cdot\rangle$ is indeed a distribution. Next, we prove the asserted formula. For this, we note the following elementary equality

$$
\sigma_{-1} \mathcal{L}_{\varrho} \varphi=\mathcal{L}_{\varrho} e^{2 \varrho m} \sigma_{-1} \varphi
$$

for $\varphi \in L_{\varrho}^{2}(\mathbb{R} ; H)$. Let $f \in H_{\varrho}^{\alpha}(\mathbb{R} ; H)$ and compute

$$
\begin{aligned}
\langle f, \varphi\rangle & =\left\langle\mathcal{L}_{\varrho} f, \mathcal{L}_{-\varrho} \varphi\right\rangle_{L^{2}(\mathbb{R} ; H)} \\
& =\left\langle\mathcal{L}_{\varrho} f, \sigma_{-1} \mathcal{L}_{\varrho} \sigma_{-1} \varphi\right\rangle_{L^{2}(\mathbb{R} ; H)} \\
& =\left\langle(\mathrm{i} m+\varrho)^{\alpha} \mathcal{L}_{\varrho} f,(-\mathrm{i} m+\varrho)^{-\alpha} \sigma_{-1} \mathcal{L}_{\varrho} \sigma_{-1} \varphi\right\rangle_{L^{2}(\mathbb{R} ; H)} \\
& =\left\langle(\mathrm{i} m+\varrho)^{\alpha} \mathcal{L}_{\varrho} f, \sigma_{-1}(\mathrm{i} m+\varrho)^{-\alpha} \mathcal{L}_{\varrho} \sigma_{-1} \varphi\right\rangle_{L^{2}(\mathbb{R} ; H)} \\
& =\left\langle(\mathrm{i} m+\varrho)^{\alpha} \mathcal{L}_{\varrho} f, \sigma_{-1} \mathcal{L}_{\varrho} \partial_{0, \varrho}^{-\alpha} \sigma_{-1} \varphi\right\rangle_{L^{2}(\mathbb{R} ; H)} \\
& =\left\langle(\mathrm{i} m+\varrho)^{\alpha} \mathcal{L}_{\varrho} f, \mathcal{L}_{\varrho} \mathrm{e}^{2 \varrho m} \sigma_{-1} \partial_{0, \varrho}^{-\alpha} \sigma_{-1} \varphi\right\rangle_{L^{2}(\mathbb{R} ; H)}
\end{aligned}
$$




$$
\begin{aligned}
& =\left\langle(\mathrm{i} m+\varrho)^{\alpha} \mathcal{L}_{\varrho} f,(\mathrm{i} m+\varrho)^{\alpha} \mathcal{L}_{\varrho} \partial_{0, \varrho}^{-\alpha} \mathrm{e}^{2 \varrho m} \sigma_{-1} \partial_{0, \varrho}^{-\alpha} \sigma_{-1} \varphi\right\rangle_{L^{2}(\mathbb{R} ; H)} \\
& =\left\langle f, \partial_{0, \varrho}^{-\alpha} \mathrm{e}^{2 \varrho m} \sigma_{-1} \partial_{0, \varrho}^{-\alpha} \sigma_{-1} \varphi\right\rangle_{\varrho, \alpha}
\end{aligned}
$$

for each $\varphi \in C_{c}^{\infty}(\mathbb{R} ; H)$. In particular, in the case $\alpha=0$ we obtain

$$
\langle f, \varphi\rangle=\left\langle f, \mathrm{e}^{2 \varrho m} \varphi\right\rangle_{\varrho, 0}=\int_{\mathbb{R}}\langle f(t), \varphi(t)\rangle_{H} \mathrm{~d} t .
$$

Remark 3.5 The latter proposition shows that $\bigcup_{\varrho \neq 0, \alpha \in \mathbb{R}} H_{\varrho}^{\alpha}(\mathbb{R} ; H) \subseteq \mathcal{D}(\mathbb{R} ; H)^{\prime}$. In particular, the support of $f \in H_{\varrho}^{\alpha}(\mathbb{R} ; H)$ is then well-defined by

$$
\bigcap\left\{\mathbb{R} \backslash U ; U \subseteq \mathbb{R} \text { open, } \forall \varphi \in C_{c}^{\infty}(U ; H):\langle f, \varphi\rangle=0\right\},
$$

and the second part of the latter proposition shows, that it coincides with the usual $L^{2}$-support if $\alpha \geq 0$. Moreover, we can now compare elements in $H_{\varrho}^{\alpha}(\mathbb{R} ; H)$ and $H_{\mu}^{\beta}(\mathbb{R} ; H)$ by saying that those elements are equal if they are equal as distributions. We shall further elaborate on this matter in Proposition 3.9. In particular, we shall show that $f \mapsto\langle f, \cdot\rangle$ is injective. We shall also mention that the notation $\langle f, \varphi\rangle$ is justified, as it does not depend on $\varrho$ nor $\alpha$.

Example 3.6 Let $f \in L_{\varrho}^{2}(\mathbb{R} ; H)$. Then, by definition, $\partial_{0, \varrho} f \in H_{\varrho}^{-1}(\mathbb{R} ; H)$. We shall compute the action of $\partial_{0, \varrho} f$ as a distribution. For this let $\varphi \in C_{c}^{\infty}(\mathbb{R} ; H)$ and we compute with the formula outlined in Proposition 3.4 for $\alpha=-1$ :

$$
\begin{aligned}
\left\langle\partial_{0, \varrho} f, \varphi\right\rangle & =\left\langle\partial_{0, \varrho} f, \partial_{0, \varrho} \mathrm{e}^{2 \varrho m} \sigma_{-1} \partial_{0, \varrho} \sigma_{-1} \varphi\right\rangle_{\varrho,-1} \\
& =\left\langle(\mathrm{i} m+\varrho)^{-1} \mathcal{L}_{\varrho} \partial_{0, \varrho} f,(\mathrm{i} m+\varrho)^{-1} \mathcal{L}_{\varrho} \partial_{0, \varrho} \mathrm{e}^{2 \varrho m} \sigma_{-1} \partial_{0, \varrho} \sigma_{-1} \varphi\right\rangle_{L^{2}(\mathbb{R} ; H)} \\
& =\left\langle\mathcal{L}_{\varrho} f, \mathcal{L}_{\varrho} \mathrm{e}^{2 \varrho m} \sigma_{-1} \partial_{0, \varrho} \sigma_{-1} \varphi\right\rangle_{L^{2}(\mathbb{R} ; H)} \\
& =-\left\langle\mathcal{L}_{\varrho} f, \mathcal{L}_{\varrho} \mathrm{e}^{2 \varrho m} \partial_{0, \varrho} \varphi\right\rangle_{L^{2}(\mathbb{R} ; H)} \\
& =-\left\langle f, \mathrm{e}^{2 \varrho m} \varphi^{\prime}\right\rangle_{L_{\varrho}^{2}(\mathbb{R} ; H)} \\
& =-\int_{\mathbb{R}}\left\langle f(t), \varphi^{\prime}(t)\right\rangle_{H} \mathrm{~d} t .
\end{aligned}
$$

Thus, $\partial_{0, \varrho} f$ coincides with the distributional derivative of $L_{\varrho}^{2}(\mathbb{R} ; H)$ functions.

Lemma 3.7 Let $\alpha \in \mathbb{R}$. Let $H_{\varrho}^{\infty}(\mathbb{R} ; H):=\bigcap_{k \in \mathbb{N}} H_{\varrho}^{k}(\mathbb{R} ; H)$ for $\varrho \neq 0$.

(a) Let $\varphi \in C_{c}^{\infty}(\mathbb{R} ; H)$. For all $\varrho>0$ we have $\partial_{\varrho}^{\alpha} \varphi \in C^{\infty}(\mathbb{R} ; H) \cap H_{\varrho}^{\infty}(\mathbb{R} ; H)$ and $\inf \operatorname{spt} \partial_{\varrho}^{\alpha} \varphi \geq \inf \operatorname{spt} \varphi$. For $\varrho, \mu>0, \alpha \in \mathbb{R}$ we have $\partial_{0, \varrho}^{\alpha} \varphi=\partial_{0, \mu}^{\alpha} \varphi$.

(b) Let $\alpha \in \mathbb{R}$ and $\mu, \varrho \neq 0$. Let $\psi \in C^{\infty}(\mathbb{R} ; H) \cap H_{\varrho}^{\infty}(\mathbb{R} ; H) \cap H_{\mu}^{\infty}(\mathbb{R} ; H)$. Then there is $\left(\varphi_{n}\right)_{n \in \mathbb{N}} \in C_{c}^{\infty}(\mathbb{R} ; H)^{\mathbb{N}}$ s.t. $\varphi_{n} \rightarrow \psi$ for $n \rightarrow \infty$ in $H_{\varrho}^{\alpha}(\mathbb{R} ; H)$ and $H_{\mu}^{\alpha}(\mathbb{R} ; H)$ and $\operatorname{spt}\left(\varphi_{n}\right) \subseteq \operatorname{spt}(\psi)$ for $n \in \mathbb{N}$.

Proof (a): Let $\alpha \in \mathbb{R}$. Let $\mu, \varrho>0$. For $\alpha>0$ it holds that $\partial_{\varrho}^{\alpha}=\partial_{\varrho}^{\alpha-\lceil\alpha\rceil} \partial_{\varrho}^{\lceil\alpha\rceil}$ and $\partial_{\varrho}^{\lceil\alpha\rceil} \varphi=$ $\varphi^{(\lceil\alpha\rceil)}=\partial_{\mu}^{\lceil\alpha\rceil} \varphi \in C_{c}^{\infty}(\mathbb{R} ; H)$ and $\alpha-\lceil\alpha\rceil<0$. Thus we may assume that $\alpha<0$. By Theorem 2.6 we have $\partial_{0, \varrho}^{\alpha} \varphi=\partial_{0, \mu}^{\alpha} \varphi$ and inf spt $\partial_{0, \varrho}^{\alpha} \varphi>-\infty$. From $\varphi \in H_{\varrho}^{\infty}(\mathbb{R} ; H)$ we deduce $\partial_{0, \varrho}^{\alpha} \varphi \in H_{\varrho}^{\infty}(\mathbb{R} ; H)$.

(b): Let $k \in \mathbb{N}$ with $k \geq \alpha$. We choose a sequence $\left(\chi_{n}\right)_{n \in \mathbb{N}}$ in $C_{c}^{\infty}(\mathbb{R})$ such that spt $\chi_{n} \subseteq$ $[-n-1, n+1], \chi_{n}=1$ on $[-n, n]$ and

$$
\sup \left\{\left\|\chi_{n}^{(j)}\right\|_{\infty} ; j \in\{0, \ldots, k\}, n \in \mathbb{N}\right\}<\infty \text {. }
$$


Set $\varphi_{n}:=\chi_{n} \psi \in C_{c}^{\infty}(\mathbb{R} ; H)$. Then $\operatorname{spt}\left(\varphi_{n}\right) \subseteq \operatorname{spt}(\psi)$ for $n \in \mathbb{N}$. Since $H_{v}^{k}(\mathbb{R} ; H)$ is dense and continuously embedded into $H_{v}^{\alpha}(\mathbb{R} ; H)(v \neq 0)$, it suffices to show that $\varphi_{n} \rightarrow \psi(n \rightarrow \infty)$ in $H_{\varrho}^{k}(\mathbb{R} ; H)$ and $H_{\mu}^{k}(\mathbb{R} ; H)$. Indeed, by the product rule, the choice of $\chi_{n}$ and dominated convergence we obtain

$$
\varphi_{n}^{(k)}=\sum_{j=0}^{k}\left(\begin{array}{l}
k \\
j
\end{array}\right) \chi_{n}^{(j)} \psi^{(k-j)}=\chi_{n} \psi^{(k)}+\sum_{j=1}^{k}\left(\begin{array}{l}
k \\
j
\end{array}\right) \chi_{n}^{(j)} \psi^{(k-j)} \rightarrow \psi^{(k)}
$$

for $n \rightarrow \infty$ in $L_{\varrho}^{2}(\mathbb{R} ; H)$ and in $L_{\mu}^{2}(\mathbb{R} ; H)$.

Lemma 3.8 Let $\varrho \neq 0$ and $\alpha \in \mathbb{R}$. Then $C_{c}^{\infty}(\mathbb{R} ; H)$ is dense in $H_{\varrho}^{\alpha}(\mathbb{R} ; H)$.

Proof. Let $f \in H_{\varrho}^{\alpha}(\mathbb{R} ; H)$. We first note that it suffices to prove the assertion for $\varrho>0$, since the operator $\sigma_{-1}$ from Lemma 3.3 leaves $C_{c}^{\infty}(\mathbb{R} ; H)$ invariant. It is well known that $C_{c}^{\infty}(\mathbb{R} ; H)$ is dense in $L_{\varrho}^{2}(\mathbb{R} ; H)$. We have $\partial_{0, \varrho}^{\alpha} f \in L_{\varrho}^{2}(\mathbb{R} ; H)$. Let $\left(\psi_{n}\right)_{n \in \mathbb{N}} \in$ $C_{c}^{\infty}(\mathbb{R} ; H)^{\mathbb{N}}$ with $\psi_{n} \rightarrow \partial_{0, \varrho}^{\alpha} f(n \rightarrow \infty)$ in $L_{\varrho}^{2}(\mathbb{R} ; H)$. By Lemma 3.7(a) we have $\partial_{0, \varrho}^{-\alpha} \psi_{n} \in C^{\infty}(\mathbb{R} ; H) \cap H_{\varrho}^{\infty}(\mathbb{R} ; H)$ and by Lemma 3.7(b) we find $\left(\varphi_{n}\right)_{n \in \mathbb{N}} \in C_{c}^{\infty}(\mathbb{R} ; H)^{\mathbb{N}}$ with $\left\|\partial_{0, \varrho}^{-\alpha} \psi_{n}-\varphi_{n}\right\|_{\varrho, \alpha} \rightarrow 0(n \rightarrow \infty)$. Then

$$
\left\|f-\varphi_{n}\right\|_{\varrho, \alpha} \leq\left\|f-\partial_{0, \varrho}^{-\alpha} \psi_{n}\right\|_{\varrho, \alpha}+\left\|\partial_{0, \varrho}^{-\alpha} \psi_{n}-\varphi_{n}\right\|_{\varrho, \alpha} \rightarrow 0 \quad(n \rightarrow \infty) .
$$

With this result at hand, we can characterize those distributions, which belong to $H_{\varrho}^{\alpha}(\mathbb{R} ; H)$ for some $\alpha \in \mathbb{R}, \varrho \neq 0$, in the following way.

Proposition 3.9 Let $\psi \in \mathcal{D}(\mathbb{R} ; H)^{\prime}$ and $\alpha \in \mathbb{R}, \varrho \neq 0$. Then, there exists $f \in H_{\varrho}^{\alpha}(\mathbb{R} ; H)$ such that

$$
\psi(\varphi)=\langle f, \varphi\rangle \quad\left(\varphi \in C_{c}^{\infty}(\mathbb{R} ; H)\right)
$$

in the sense of Proposition 3.4 if and only if there is $C \geq 0$ such that

$$
|\psi(\varphi)| \leq C\|\varphi\|_{-\varrho,-\alpha}
$$

for each $\varphi \in C_{c}^{\infty}(\mathbb{R} ; H)$.

Proof. Assume first that there is $f \in H_{\varrho}^{\alpha}(\mathbb{R} ; H)$ representing $\psi$. Then we estimate

$$
\begin{aligned}
|\psi(\varphi)| & =|\langle f, \varphi\rangle| \\
& =\left|\int_{\mathbb{R}}\left\langle\mathcal{L}_{\varrho} f(t), \mathcal{L}_{-\varrho} \varphi(t)\right\rangle_{H} \mathrm{~d} t\right| \\
& =\left|\int_{\mathbb{R}}\left\langle(\mathrm{i} t+\varrho)^{\alpha} \mathcal{L}_{\varrho} f(t),(-\mathrm{i} t+\varrho)^{-\alpha} \mathcal{L}_{-\varrho} \varphi(t)\right\rangle_{H} \mathrm{~d} t\right| \\
& \leq\left\|\mathcal{L}_{\varrho} f\right\|_{H^{\alpha}(\mathrm{i} m+\varrho)}\left\|\mathcal{L}_{-\varrho} \varphi\right\|_{H^{-\alpha}(\mathrm{i} m-\varrho)} \\
& =\|f\|_{\varrho, \alpha}\|\varphi\|_{-\varrho,-\alpha}
\end{aligned}
$$

for each $\varphi \in C_{c}^{\infty}(\mathbb{R} ; H)$. Let $C \geq 0$ such that $\psi$ satisfies for $\varphi \in C_{c}^{\infty}(\mathbb{R} ; H)$

$$
|\psi(\varphi)| \leq C\|\varphi\|_{-\varrho,-\alpha} .
$$


The operator $A:=\partial_{0, \varrho}^{-\alpha} \mathrm{e}^{2 \varrho m} \sigma_{-1} \partial_{0, \varrho}^{-\alpha} \sigma_{-1}: H_{-\varrho}^{-\alpha}(\mathbb{R} ; H) \rightarrow H_{\varrho}^{\alpha}(\mathbb{R} ; H)$ (cf. Proposition 3.4) is unitary. Thus for $\varphi \in C_{c}^{\infty}(\mathbb{R} ; H)$

$$
\left|\psi\left(A^{-1} \varphi\right)\right| \leq C\left\|A^{-1} \varphi\right\|_{-\varrho,-\alpha}=C\|\varphi\|_{\varrho, \alpha} .
$$

Moreover, $C_{c}^{\infty}(\mathbb{R} ; H) \subseteq H_{\varrho}^{\alpha}(\mathbb{R} ; H)$ is dense. Thus $\psi\left(A^{-1} \cdot\right)$ can be extended continuously to $H_{\varrho}^{\alpha}(\mathbb{R} ; H)$. By the Riesz representation theorem, there is a $f \in H_{\varrho}^{\alpha}(\mathbb{R} ; H)$ such that for $\varphi \in H_{\varrho}^{\alpha}(\mathbb{R} ; H)$

$$
\psi\left(A^{-1} \varphi\right)=\langle f, \varphi\rangle_{\varrho, \alpha} .
$$

By Theorem 3.4 we have for $\varphi \in C_{c}^{\infty}(\mathbb{R} ; H)$

$$
\psi(\varphi)=\psi\left(A^{-1} A \varphi\right)=\langle f, A \varphi\rangle_{\varrho, \alpha}=\langle f, \varphi\rangle .
$$

In the next proposition, we shall also obtain the announced uniqueness statement, that is, the injectivity of the mapping $f \mapsto\langle f, \cdot\rangle$.

Proposition 3.10 Let $\alpha \in \mathbb{R}$ and $\mu, \varrho>0$. Moreover, let $f \in H_{\varrho}^{\alpha}(\mathbb{R} ; H)$ and $g \in H_{\mu}^{\alpha}(\mathbb{R} ; H)$. Then the following statements are equivalent:

(i) $f=g$ in the sense of distributions, i.e., for each $\varphi \in C_{c}^{\infty}(\mathbb{R} ; H)$ we have that

$$
\int_{\mathbb{R}}\left\langle\mathcal{L}_{\varrho} f(t), \mathcal{L}_{-\varrho} \varphi(t)\right\rangle_{H} \mathrm{~d} t=\int_{\mathbb{R}}\left\langle\mathcal{L}_{\mu} g(t), \mathcal{L}_{-\mu} \varphi(t)\right\rangle_{H} \mathrm{~d} t .
$$

(ii) $\partial_{0, \varrho}^{\alpha} f=\partial_{0, \mu}^{\alpha} g$ as functions in $L_{\mathrm{loc}}^{1}(\mathbb{R} ; H)$.

(iii) There is a sequence $\left(\varphi_{n}\right)_{n \in \mathbb{N}}$ in $C_{c}^{\infty}(\mathbb{R} ; H)$ with $\varphi_{n} \rightarrow f$ in $H_{\varrho}^{\alpha}(\mathbb{R} ; H)$ and $\varphi_{n} \rightarrow g$ in $H_{\mu}^{\alpha}(\mathbb{R} ; H)$ as $n \rightarrow \infty$.

Proof (i) $\Rightarrow$ (ii): Let $\psi \in C_{c}^{\infty}(\mathbb{R} ; H)$ and $\widetilde{\psi}:=\sigma_{-1} \partial_{\varrho}^{\alpha} \sigma_{-1} \psi=\sigma_{-1} \partial_{\mu}^{\alpha} \sigma_{-1} \psi$. Then by Lemma 3.7(a) $\widetilde{\psi} \in C^{\infty}(\mathbb{R} ; H) \cap H_{-\varrho}^{\infty}(\mathbb{R} ; H) \cap H_{-\mu}^{\infty}(\mathbb{R} ; H)$. By Lemma 3.7(b) there is $\left(\varphi_{n}\right)_{n \in \mathbb{N}} \in$ $C_{c}^{\infty}(\mathbb{R} ; H)^{\mathbb{N}}$ with $\varphi_{n} \rightarrow \widetilde{\psi}(n \rightarrow \infty)$ in $H_{-\varrho}^{-\alpha}(\mathbb{R} ; H)$ and in $H_{-\mu}^{-\alpha}(\mathbb{R} ; H)$. Thus

$$
\begin{aligned}
\int_{\mathbb{R}}\left\langle\partial_{0, \varrho}^{\alpha} f(t), \psi(t)\right\rangle_{H} \mathrm{~d} t & =\left\langle\partial_{0, \varrho}^{\alpha} f, \mathrm{e}^{2 \varrho m} \psi\right\rangle_{\varrho, 0} \\
& =\left\langle f, \partial_{0, \varrho}^{-\alpha} \mathrm{e}^{2 \varrho m} \sigma_{-1} \partial_{0, \varrho}^{-\alpha} \sigma_{-1}\left(\sigma_{-1} \partial_{0, \varrho}^{\alpha} \sigma_{-1} \psi\right)\right\rangle_{\varrho, \alpha} \\
& =\lim _{n \rightarrow \infty}\left\langle f, \partial_{0, \varrho}^{-\alpha} \mathrm{e}^{2 \varrho m} \sigma_{-1} \partial_{0, \varrho}^{-\alpha} \sigma_{-1} \varphi_{n}\right\rangle_{\varrho, \alpha} \\
& =\lim _{n \rightarrow \infty}\left\langle f, \varphi_{n}\right\rangle \\
& =\lim _{n \rightarrow \infty}\left\langle g, \varphi_{n}\right\rangle \\
& =\lim _{n \rightarrow \infty}\left\langle f, \partial_{0, \mu}^{-\alpha} \mathrm{e}^{2 \mu m} \sigma_{-1} \partial_{0, \mu}^{-\alpha} \sigma_{-1} \varphi_{n}\right\rangle_{\mu, \alpha} \\
& =\int_{\mathbb{R}}\left\langle\partial_{0, \mu}^{\alpha} f(t), \psi(t)\right\rangle_{H} \mathrm{~d} t .
\end{aligned}
$$

(ii) $\Rightarrow$ (iii): Define $\tilde{f}_{n}:=\chi_{[-n, n]} \cdot \partial_{0, \varrho}^{\alpha} f=\chi_{[-n, n]} \cdot \partial_{0, \mu}^{\alpha} g$ for $n \in \mathbb{N}$. Without loss of generality let $\varrho<\mu$. Take a function $\psi_{n} \in C_{c}^{\infty}(\mathbb{R} ; H)$ with spt $\psi_{n} \subseteq[-n, n]$ such that

$$
\left\|\tilde{f}_{n}-\psi_{n}\right\|_{\varrho, 0} \leq \frac{1}{n} \mathrm{e}^{(\varrho-\mu) n} .
$$


Then, we estimate

$$
\begin{aligned}
\left\|\widetilde{f}_{n}-\psi_{n}\right\|_{\mu, 0}^{2} & =\int_{-n}^{n}\left\|\widetilde{f}_{n}(t)-\psi_{n}(t)\right\|_{H}^{2} \mathrm{e}^{-2 \mu t} \mathrm{~d} t \\
& =\int_{-n}^{n}\left\|\widetilde{f}_{n}(t)-\psi_{n}(t)\right\|_{H}^{2} \mathrm{e}^{-2 \varrho t} \mathrm{e}^{2(\varrho-\mu) t} \mathrm{~d} t \\
& \leq\left\|\widetilde{f}_{n}-\psi_{n}\right\|_{\varrho, 0}^{2} \mathrm{e}^{2(\mu-\varrho) n} \leq \frac{1}{n^{2}} .
\end{aligned}
$$

Hence $\psi_{n} \rightarrow \partial_{0, \varrho}^{\alpha} f=\partial_{0, \mu}^{\alpha} g$ in $L_{\varrho}^{2}(\mathbb{R} ; H)$ and in $L_{\mu}^{2}(\mathbb{R} ; H)$ by the triangle inequality and dominated convergence. We set $\widetilde{\varphi}_{n}:=\partial_{0, \varrho}^{-\alpha} \psi_{n}=\partial_{0, \mu}^{-\alpha} \psi_{n} \in C^{\infty}(\mathbb{R} ; H) \cap H_{\varrho}^{\infty}(\mathbb{R} ; H) \cap$ $H_{\mu}^{\infty}(\mathbb{R} ; H)$. Then $\widetilde{\varphi}_{n} \rightarrow f$ and $\widetilde{\varphi}_{n} \rightarrow g$ in $H_{\varrho}^{\alpha}(\mathbb{R} ; H)$ and in $H_{\mu}^{\alpha}(\mathbb{R} ; H)$ respectively. We use Lemma 3.7(b) and choose a sequence $\left(\varphi_{n}\right)_{n \in \mathbb{N}} \in C_{c}^{\infty}(\mathbb{R} ; H)^{\mathbb{N}}$ with $\left\|\widetilde{\varphi}_{n}-\varphi_{n}\right\|_{\varrho, \alpha} \rightarrow 0$. Then

$$
\begin{array}{rlrl}
\left\|f-\varphi_{n}\right\|_{\varrho, \alpha} \leq\left\|f-\widetilde{\varphi}_{n}\right\|_{\varrho, \alpha}+\left\|\widetilde{\varphi}_{n}-\varphi_{n}\right\|_{\varrho, \alpha} \rightarrow 0 & & (n \rightarrow \infty), \\
\left\|g-\varphi_{n}\right\|_{\mu, \alpha} \leq\left\|g-\widetilde{\varphi}_{n}\right\|_{\mu, \alpha}+\left\|\widetilde{\varphi}_{n}-\varphi_{n}\right\|_{\mu, \alpha} \rightarrow 0 & (n \rightarrow \infty) .
\end{array}
$$

(iii) $\Rightarrow$ (i): Let $\left(\varphi_{n}\right)_{n \in \mathbb{N}}$ be a sequence in $C_{c}^{\infty}(\mathbb{R} ; H)$ such that $\varphi_{n} \rightarrow f$ and $\varphi_{n} \rightarrow g$ in $H_{\varrho}^{\alpha}(\mathbb{R} ; H)$ and $H_{\mu}^{\alpha}(\mathbb{R} ; H)$, respectively. Let $\varphi \in C_{c}^{\infty}(\mathbb{R} ; H)$. Then we have according to Proposition 3.4

$$
\begin{aligned}
\int_{\mathbb{R}}\left\langle\mathcal{L}_{\varrho} f(t), \mathcal{L}_{-\varrho} \varphi(t)\right\rangle_{H} \mathrm{~d} t & =\langle f, \varphi\rangle \\
& =\left\langle f, \partial_{0, \varrho}^{-\alpha} \mathrm{e}^{2 \varrho m} \sigma_{-1} \partial_{0, \varrho}^{-\alpha} \sigma_{-1} \varphi\right\rangle_{\varrho, \alpha} \\
& =\lim _{n \rightarrow \infty}\left\langle\varphi_{n}, \partial_{0, \varrho}^{-\alpha} \mathrm{e}^{2 \varrho m} \sigma_{-1} \partial_{0, \varrho}^{-\alpha} \sigma_{-1} \varphi\right\rangle_{\varrho, \alpha} \\
& =\lim _{n \rightarrow \infty}\left\langle\varphi_{n}, \varphi\right\rangle \\
& =\lim _{n \rightarrow \infty}\left\langle\varphi_{n}, \partial_{0, \mu}^{-\alpha} \mathrm{e}^{2 \mu m} \sigma_{-1} \partial_{0, \mu}^{-\alpha} \sigma_{-1} \varphi\right\rangle_{\mu, \alpha} \\
& =\langle g, \varphi\rangle, \\
& =\int_{\mathbb{R}}\left\langle\mathcal{L}_{\mu} f(t), \mathcal{L}_{-\mu} \varphi(t)\right\rangle \mathrm{d} t
\end{aligned}
$$

which completes the proof.

\section{A Unified Solution Theory-Well-Posedness and Causality of Fractional Differential Equations}

We are now able to study abstract fractional differential equations of the form

$$
\partial_{0, \varrho}^{\alpha} u=F(u) .
$$

In order to obtain well-posedness of the latter problem, we need to restrict the class of admissible right-hand sides $F$ in the latter equation. 
Definition Let $\varrho_{0}>0$ and $\beta, \gamma \in \mathbb{R}$. We call a function $F: \operatorname{dom}(F) \subseteq \bigcap_{\varrho \geq \varrho_{0}} H_{\varrho}^{\beta}(\mathbb{R} ; H) \rightarrow$ $\bigcap_{\varrho \geq \varrho_{0}} H_{\varrho}^{\gamma}(\mathbb{R} ; H)$ eventually $(\beta, \gamma)$-Lipschitz continuous, if $\operatorname{dom}(F) \supseteq C_{c}^{\infty}(\mathbb{R} ; H)$ and there exists $v \geq \varrho_{0}$ such that for each $\varrho \geq v$ the function $F$ has a Lipschitz continuous extension

$$
F_{\varrho}: H_{\varrho}^{\beta}(\mathbb{R} ; H) \rightarrow H_{\varrho}^{\gamma}(\mathbb{R} ; H)
$$

satisfying $\sup _{\varrho \geq v}\left|F_{\varrho}\right|_{\text {Lip }}<\infty$. Moreover, we call $F$ eventually $(\beta, \gamma)$-contracting, if $F$ is eventually $(\beta, \gamma)$-Lipschitz continuous and $\lim \sup _{\varrho \rightarrow \infty}\left|F_{\varrho}\right|_{\text {Lip }}<1$. Here, we denote by $|\cdot|_{\text {Lip }}$ the smallest Lipschitz constant of a Lipschitz continuous function:

$$
\left|F_{\varrho}\right|_{\text {lip }}:=\sup _{f, g \in H_{\varrho}^{\beta}(\mathbb{R} ; H), f \neq g} \frac{\left\|F_{\varrho}(f)-F_{\varrho}(g)\right\|_{\varrho, \gamma}}{\|f-g\|_{\varrho, \beta}} .
$$

Note that by Lemma 3.8, any eventually Lipschitz continuous function is densely defined. Thus, the Lipschitz continuous extension $F_{\varrho}$ is unique.

Remark 4.1 (a) If $f \in H_{\varrho}^{\beta}(\mathbb{R} ; H)$ and $g \in H_{\mu}^{\beta}$ (R्R;H) generate the same distribution, we have that

$$
F_{\varrho}(f)=F_{\mu}(g)
$$

Indeed, by Proposition 3.10 there exists a sequence $\left(\varphi_{n}\right)_{n \in \mathbb{N}}$ in $C_{c}^{\infty}(\mathbb{R} ; H)$ with $\varphi_{n} \rightarrow f$ and $\varphi_{n} \rightarrow g$ in $H_{\varrho}^{\beta}(\mathbb{R} ; H)$ and $H_{\mu}^{\beta}(\mathbb{R} ; H)$, respectively. We infer that

$$
F_{\varrho}(f)=\lim _{n \rightarrow \infty} F\left(\varphi_{n}\right) \quad \text { and } \quad F_{\mu}(g)=\lim _{n \rightarrow \infty} F\left(\varphi_{n}\right)
$$

with convergence in $H_{\varrho}^{\gamma}(\mathbb{R} ; H)$ and $H_{\mu}^{\gamma}(\mathbb{R} ; H)$ respectively. Consequently

$$
\partial_{0, \varrho}^{\gamma} F_{\varrho}(f) \leftarrow \partial_{0, \varrho}^{\gamma} F\left(\varphi_{n}\right)=\partial_{0, \mu}^{\gamma} F\left(\varphi_{n}\right) \rightarrow \partial_{0, \mu}^{\gamma} F_{\mu}(g)
$$

with convergence in $L_{\varrho}^{2}(\mathbb{R} ; H)$ and hence almost everywhere for a suitable subsequence of $\left(\varphi_{n}\right)_{n \in \mathbb{N}}$. The assertion follows from Proposition 3.10.

(b) We shall need the following elementary observation later on. Let $F$ be evenutally $(\beta, \gamma)$-Lipschitz continuous, $\alpha \in \mathbb{R}$. Let $\varrho \geq \varrho_{0}$. Then

$$
\widetilde{F}: C_{c}^{\infty}(\mathbb{R} ; H) \ni \varphi \mapsto F_{\varrho}\left(\partial_{0, \varrho}^{\alpha} \varphi\right)
$$

is eventually $(\beta+\alpha, \gamma)$-Lipschitz continuous. Indeed, the assertion follows from part (a) and

$$
\|\widetilde{F}(f)-\widetilde{F}(g)\|_{\mu, \gamma} \leq\left|F_{\mu}\right|_{\text {Lip }}\left\|\partial_{0, \mu}^{\alpha} f-\partial_{0, \mu}^{\alpha} g\right\|_{\mu, \beta}=\left|F_{\mu}\right|_{\text {Lip }}\|f-g\|_{\mu, \alpha+\beta},
$$

for $\mu \geq v, f, g \in C_{c}^{\infty}(\mathbb{R} ; H)$.

Theorem 4.2 Let $\alpha>0, \beta \in \mathbb{R}, \varrho_{0}>0$ and $F: \operatorname{dom}(F) \subseteq \bigcap_{\varrho \geq \varrho_{0}} H_{\varrho}^{\beta}(\mathbb{R} ; H) \rightarrow$ $\bigcap_{\varrho \geq \varrho_{0}} H_{\varrho}^{\beta-\alpha}(\mathbb{R} ; H)$ be eventually $(\beta, \beta-\alpha)$-contracting. Then there exists $v \geq \varrho_{0}$ such that for each $\varrho \geq v$ there is a unique $u_{\varrho} \in H_{\varrho}^{\beta}(\mathbb{R} ; H)$ satisfying

$$
\partial_{0, \varrho}^{\alpha} u_{\varrho}=F_{\varrho}\left(u_{\varrho}\right) .
$$


Proof This is a simple consequence of the contraction mapping theorem. Indeed, choosing $v \geq \varrho_{0}$ large enough, such that $\left|F_{\varrho}\right|_{\text {Lip }}<1$ for each $\varrho \geq v$, we obtain that

$$
\partial_{0, \varrho}^{-\alpha} F_{\varrho}: H_{\varrho}^{\beta}(\mathbb{R} ; H) \rightarrow H_{\varrho}^{\beta}(\mathbb{R} ; H)
$$

is a strict contraction, since $\partial_{0, \varrho}^{-\alpha}: H_{\varrho}^{\beta-\alpha}(\mathbb{R} ; H) \rightarrow H_{\varrho}^{\beta}(\mathbb{R} ; H)$ is unitary by Lemma 3.2. Hence, the mapping $\partial_{0, \varrho}^{-\alpha} F_{\varrho}$ admits a unique fixed point $u_{\varrho} \in H_{\varrho}^{\beta}(\mathbb{R} ; H)$, which is equivalent to $u_{\varrho}$ being a solution of (4.1).

Corollary 4.3 Let $\alpha>0, \beta \in \mathbb{R}, \varrho_{0}>0$ and $F: \operatorname{dom}(F) \subseteq \bigcap_{\varrho \geq \varrho_{0}} H_{\varrho}^{\beta}(\mathbb{R} ; H) \rightarrow$ $\bigcap_{\varrho \geq \varrho_{0}} H_{\varrho}^{\beta-\gamma}(\mathbb{R} ; H)$ for some $\gamma \in[0, \alpha[$ be eventually $(\beta, \beta-\gamma)$-Lipschitz continuous. Then there exists $v \geq \varrho_{0}$ such that for each $\varrho \geq v$ there is a unique $u_{\varrho} \in H_{\varrho}^{\beta}(\mathbb{R} ; H)$ satisfying

$$
\partial_{0, \varrho}^{\alpha} u_{\varrho}=F_{\varrho}\left(u_{\varrho}\right) .
$$

Proof. It suffices to prove that $\iota_{\beta-\gamma \rightarrow \beta-\alpha} \circ F$ is eventually $(\beta, \beta-\alpha)$-contracting by Theorem 4.2. Let $v \geq \varrho$, s.t. for $\varrho \geq v, F_{\varrho}$ exists. Then for $\varrho \geq v$

$$
\left|\iota_{\beta-\gamma \rightarrow \beta-\alpha} \circ F_{\varrho}\right|_{\text {Lip }} \leq\left\|\iota_{\beta-\gamma \rightarrow \beta-\alpha}\right\|\left|F_{\varrho}\right|_{\text {Lip }} \leq \varrho^{\gamma-\alpha}\left|F_{\varrho}\right|_{\text {Lip }}
$$

by Lemma 3.2. Since $\left|F_{\varrho}\right|_{\text {Lip }}$ is bounded in $\varrho$ on $[v, \infty$ [ by assumption, we infer

$$
\limsup _{\varrho \rightarrow \infty}\left|\iota_{\beta-\gamma \rightarrow \beta-\alpha} \circ F_{\varrho}\right|_{\text {Lip }}=0<1 \text {. }
$$

Next, we want to show that the solution $u_{\varrho}$ of (4.1) is actually independent of the particular choice of $\varrho$. For doing so, we need the concept of causality, which will be addressed in the next propositions.

Lemma 4.4 Let $\varrho>0, \alpha \in \mathbb{R}$ and $a \in \mathbb{R}$. Let $f \in H_{\varrho}^{\alpha}(\mathbb{R} ; H)$ with spt $f \subseteq \mathbb{R}_{\geq a}$. Then there is a sequence $\left(\varphi_{n}\right)_{n \in \mathbb{N}} \in C_{c}^{\infty}(\mathbb{R} ; H)^{\mathbb{N}}$ with $\operatorname{spt} \varphi_{n} \subseteq \mathbb{R}_{\geq a}$ for $n \in \mathbb{N}$ and $\varphi_{n} \rightarrow f$ in $H_{\varrho}^{\alpha}(\mathbb{R} ; H)$ as $n \rightarrow \infty$.

Proof. Let $\left(\widetilde{\psi}_{n}\right)_{n \in \mathbb{N}} \in C_{c}^{\infty}(\mathbb{R} ; H)^{\mathbb{N}}$ be such that $\widetilde{\psi}_{n} \rightarrow \partial_{0, \varrho}^{\alpha} f$ in $H_{\varrho}^{0}(\mathbb{R} ; H)$ as $n \rightarrow \infty$. We may assume that spt $\widetilde{\psi}_{n} \subseteq \mathbb{R}_{>a}$. We set $\psi_{n}:=\partial_{0, \varrho}^{-\alpha} \widetilde{\psi}_{n}$ for $n \in \mathbb{N}$. Then $\psi_{n} \rightarrow f$ as $n \rightarrow \infty$ in $H_{\varrho}^{\alpha}(\mathbb{R} ; H)$ and inf spt $\psi_{n}>a$ by Lemma 3.7(a). We use Lemma 3.7(b) and pick a sequence $\left(\varphi_{n}\right)_{n \in \mathbb{N}} \in C_{c}^{\infty}(\mathbb{R} ; H)^{\mathbb{N}}$ with $\operatorname{spt}\left(\varphi_{n}\right) \subseteq \operatorname{spt}\left(\psi_{n}\right)$ for $n \in \mathbb{N}$ and $\varphi_{n}-\psi_{n} \rightarrow 0$ in $H_{\varrho}^{\alpha}(\mathbb{R} ; H)$ when $n \rightarrow \infty$. Then

$$
\left\|\varphi_{n}-f\right\|_{\varrho, \alpha} \leq\left\|\varphi_{n}-\psi_{n}\right\|_{\varrho, \alpha}+\left\|\psi_{n}-f\right\|_{\varrho, \alpha} \rightarrow 0 \quad(n \rightarrow \infty) .
$$

Proposition 4.5 Let $f \in H_{\varrho}^{\alpha}(\mathbb{R} ; H)$ for some $\alpha \in \mathbb{R}, \varrho>0$. Assume that spt $f \subseteq \mathbb{R}_{\geq a}$ for some $a \in \mathbb{R}$. Then

$$
\operatorname{spt} \partial_{0, \varrho}^{\beta} f \subseteq \mathbb{R}_{\geq a}
$$

for all $\beta \in \mathbb{R}$.

Proof. Let $\varphi \in C_{c}^{\infty}(\mathbb{R} ; H)$ with spt $\varphi \subseteq \mathbb{R}_{<a}$. By Lemma 4.4 we pick a sequence $\left(\varphi_{n}\right)_{n \in \mathbb{N}} \in$ $C_{c}^{\infty}(\mathbb{R} ; H)^{\mathbb{N}}$, s.t. spt $\varphi_{n} \subseteq \mathbb{R}_{\geq a}(n \in \mathbb{N})$ and $\varphi_{n} \rightarrow f$ in $H_{\varrho}^{\alpha}(\mathbb{R} ; H)$. Then spt $\partial_{0, \varrho}^{\beta} \varphi_{n} \subseteq \mathbb{R}_{\geq a}$ by Lemma 3.7(a). By Proposition 3.4 we have

$$
\left\langle\partial_{0, \varrho}^{\beta} \varphi_{n}, \varphi\right\rangle=\int_{\mathbb{R}}\left\langle\partial_{0, \varrho}^{\beta} \varphi_{n}(t), \varphi(t)\right\rangle_{H} \mathrm{~d} t=0 .
$$


Since $\partial_{0, \varrho}^{\beta}$ is unitary, we have $\partial_{0, \varrho}^{\beta} \varphi_{n} \rightarrow \partial_{0, \varrho}^{\beta} f$ in $H_{\varrho}^{\alpha-\beta}(\mathbb{R} ; H)$. We compute

$$
\begin{aligned}
\left\langle\partial_{0, \varrho}^{\beta} f, \varphi\right\rangle & =\left\langle\partial_{0, \varrho}^{\beta} f, \partial_{0, \varrho}^{-(\alpha-\beta)} \mathrm{e}^{2 \varrho m} \sigma_{-1} \partial_{0, \varrho}^{-(\alpha-\beta)} \sigma_{-1} \varphi\right\rangle_{\varrho, \alpha-\beta} \\
& =\lim _{n \rightarrow \infty}\left\langle\partial_{0, \varrho}^{\beta} \varphi_{n}, \partial_{0, \varrho}^{-(\alpha-\beta)} \mathrm{e}^{2 \varrho m} \sigma_{-1} \partial_{0, \varrho}^{-(\alpha-\beta)} \sigma_{-1} \varphi\right\rangle_{\varrho, \alpha-\beta} \\
& =\lim _{n \rightarrow \infty}\left\langle\partial_{0, \varrho}^{\beta} \varphi_{n}, \varphi\right\rangle \\
& =0 .
\end{aligned}
$$

The proof of the following theorem outlining causality of $\partial_{0, \varrho}^{-\alpha} F_{\varrho}$, is in spirit similar to the approach in [5, Theorem 4.5]. However, one has to adopt the distributional setting and the (different) definition of eventually Lipschitz continuity here accordingly.

Theorem 4.6 Let the assumptions of Theorem 4.2 be satisfied. Then, for each $\varrho \geq v$, where $v$ is chosen according to Theorem 4.2, the mapping

$$
\partial_{0, \varrho}^{-\alpha} F_{\varrho}: H_{\varrho}^{\beta}(\mathbb{R} ; H) \rightarrow H_{\varrho}^{\beta}(\mathbb{R} ; H)
$$

is causal, that is, for each $u, v \in H_{\varrho}^{\beta}(\mathbb{R} ; H)$ satisfying $\operatorname{spt}(u-v) \subseteq \mathbb{R}_{\geq a}$ for some a $\in \mathbb{R}$, it holds that $\operatorname{spt}\left(\partial_{0, \varrho}^{-\alpha} F_{\varrho}(u)-\partial_{0, \varrho}^{-\alpha} F_{\varrho}(v)\right) \subseteq \mathbb{R}_{\geq a}$. Here, the support is meant in the sense of distributions.

Proof First of all, we shall show the result for $u, v \in C_{c}^{\infty}(\mathbb{R} ; H)$. So, let $u, v \in C_{c}^{\infty}(\mathbb{R} ; H)$ with $\operatorname{spt}(u-v) \subseteq \mathbb{R}_{\geq a}$. Take $\varphi \in C_{c}^{\infty}(\mathbb{R} ; H)$ with spt $\varphi \subseteq \mathbb{R}_{<a}$. Let $\mu \geq \varrho$. Then $F_{\varrho}(u)=F_{\mu}(u)$ and

$$
\begin{aligned}
\left\langle\partial_{0, \varrho}^{-\alpha}\left(F_{\varrho}(u)-F_{\varrho}(v)\right), \varphi\right\rangle & =\left\langle\partial_{0, \mu}^{-\alpha}\left(F_{\mu}(u)-F_{\mu}(v)\right), \varphi\right\rangle \\
& =\left\langle\partial_{0, \mu}^{-\alpha}\left(F_{\mu}(u)-F_{\mu}(v)\right), \partial_{0, \mu}^{-\beta} \mathrm{e}^{2 \mu m} \sigma_{-1} \partial_{0, \mu}^{-\beta} \sigma_{-1} \varphi\right\rangle_{\mu, \beta} \\
& =\left\langle F_{\mu}(u)-F_{\mu}(v), \partial_{0, \mu}^{-(\beta-\alpha)} \mathrm{e}^{2 \mu m} \sigma_{-1} \partial_{0, \mu}^{-\beta} \sigma_{-1} \varphi\right\rangle_{\mu, \beta-\alpha} \\
& \leq\left\|F_{\mu}(u)-F_{\mu}(v)\right\|_{\mu, \beta-\alpha}\left\|\partial_{0, \mu}^{-(\beta-\alpha)} \mathrm{e}^{2 \mu m} \sigma_{-1} \partial_{0, \mu}^{-\beta} \sigma_{-1} \varphi\right\|_{\mu, \beta-\alpha} \\
& \leq\left|F_{\mu}\right|_{\text {lip }}\|u-v\|_{\mu, \beta}\left\|\partial_{0, \mu}^{-\beta} \sigma_{-1} \varphi\right\|_{\mu, 0},
\end{aligned}
$$

where we have used that $\partial_{0, \mu}^{-(\beta-\alpha)} \mathrm{e}^{2 \mu m} \sigma_{-1}: H_{\mu}^{0}(\mathbb{R} ; H) \rightarrow H_{\mu}^{\beta-\alpha}(\mathbb{R} ; H)$ is unitary and $\varphi \in H_{-\mu}^{-\beta}(\mathbb{R} ; H)$. According to Proposition 4.5 we have that $\operatorname{spt}_{0, \mu}^{-\beta} \sigma_{-1} \varphi \subseteq \mathbb{R}_{>-a}$ and hence, we compute

$$
\begin{aligned}
& \left\|\partial_{0, \mu}^{-\beta} \sigma_{-1} \varphi\right\|_{\mu, 0}^{2} \\
& \quad=\int_{-a}^{\infty}\left\|\left(\partial_{0, \mu}^{-\beta} \sigma_{-1} \varphi\right)(t)\right\|_{H}^{2} \mathrm{e}^{-2 \mu t} \mathrm{~d} t=\int_{0}^{\infty}\left\|\left(\partial_{0, \mu}^{-\beta} \sigma_{-1} \varphi\right)(t-a)\right\|_{H}^{2} \mathrm{e}^{-2 \mu t} \mathrm{~d} t \mathrm{e}^{2 \mu a} .
\end{aligned}
$$

On the other hand 


$$
\begin{aligned}
\|u-v\|_{\mu, \beta}^{2} & =\left\|\partial_{0, \mu}^{\beta}(u-v)\right\|_{\mu, 0}^{2} \\
& =\int_{a}^{\infty}\left\|\partial_{0, \mu}^{\beta}(u-v)(t)\right\|_{H}^{2} \mathrm{e}^{-2 \mu t} \mathrm{~d} t \\
& =\int_{0}^{\infty}\left\|\partial_{0, \mu}^{\beta}(u-v)(t+a)\right\|_{H}^{2} \mathrm{e}^{-2 \mu t} \mathrm{~d} t \mathrm{e}^{-2 \mu a}
\end{aligned}
$$

and consequently,

$$
\begin{aligned}
\left|F_{\mu}\right|_{\text {Lip }}\|u-v\|_{\mu, \beta}\left\|\partial_{0, \mu}^{-\beta} \sigma_{-1} \varphi\right\|_{-\mu, 0} \\
=\left|F_{\mu}\right|_{\text {Lip }}\left(\int_{0}^{\infty}\left\|\partial_{0, \mu}^{\beta}(u-v)(t+a)\right\|_{H}^{2} \mathrm{e}^{-2 \mu t} \mathrm{~d} t\right)^{\frac{1}{2}} \\
\cdot \int_{0}^{\infty}\left\|\left(\partial_{0, \mu}^{-\beta} \sigma_{-1} \varphi\right)(t-a)\right\|_{H}^{2} \mathrm{e}^{-2 \mu t} \mathrm{~d} t \rightarrow 0 \quad(\mu \rightarrow \infty),
\end{aligned}
$$

by dominated convergence. Summarizing, we have shown that $\operatorname{spt}\left(\partial_{0, \varrho}^{-\alpha} F_{\varrho}(u)-\partial_{0, \varrho}^{-\alpha} F_{\varrho}(v)\right) \subseteq$ $\mathbb{R}_{\geq a}$ for $u, v \in C_{c}^{\infty}(\mathbb{R} ; H)$ satisfying $\operatorname{spt}(u-v) \subseteq \mathbb{R}_{\geq a}$.

Before we conclude the proof, we show that if $\left(w_{n}\right)_{n \in \mathbb{N}}$ is a convergent sequence in $H_{\varrho}^{\beta}(\mathbb{R} ; H)$ with spt $w_{n} \subseteq \mathbb{R}_{\geq a}$ for each $n \in \mathbb{N}$, then its limit $w$ also satisfies spt $w \subseteq \mathbb{R}_{\geq a}$. For doing so, let $\varphi \in C_{c}^{\infty}(\mathbb{R} ; H)$ with spt $\varphi \subseteq \mathbb{R}_{<a}$. Then

$$
\begin{aligned}
\langle w, \varphi\rangle & =\left\langle w, \partial_{0, \varrho}^{-\beta} \mathrm{e}^{2 \varrho m} \sigma_{-1} \partial_{0, \varrho}^{-\beta} \sigma_{-1} \varphi\right\rangle_{\varrho, \beta} \\
& =\lim _{n \rightarrow \infty}\left\langle w_{n}, \partial_{0, \varrho}^{-\beta} \mathrm{e}^{2 \varrho m} \sigma_{-1} \partial_{0, \varrho}^{-\beta} \sigma_{-1} \varphi\right\rangle_{\varrho, \beta}=\lim _{n \rightarrow \infty}\left\langle w_{n}, \varphi\right\rangle=0 .
\end{aligned}
$$

Finally, let $u, v \in H_{\varrho}^{\beta}(\mathbb{R} ; H)$ with $\operatorname{spt}(u-v) \subseteq \mathbb{R}_{\geq a}$ According to Lemma 4.4 there is a sequence $\left(\varphi_{n}\right)_{n \in \mathbb{N}} \in C_{c}^{\infty}(\mathbb{R} ; H)^{\mathbb{N}}$ with spt $\varphi_{n} \subseteq \mathbb{R}_{\geq a}$ and $\varphi_{n} \rightarrow u-v$ in $H_{\varrho}^{\alpha}(\mathbb{R} ; H)$ as $n \rightarrow \infty$. Let $\left(v_{n}\right)_{n \in \mathbb{N}} \in C_{c}^{\infty}(\mathbb{R} ; H)$ with $v_{n} \rightarrow v$ in $H_{\varrho}^{\alpha}(\mathbb{R} ; H)$ as $n \rightarrow \infty$. We set $u_{n}:=\varphi_{n}+v_{n}$. Then $u_{n} \rightarrow u$ in $H_{\varrho}^{\alpha}(\mathbb{R} ; H)$ and $\operatorname{spt}\left(u_{n}-v_{n}\right) \subseteq \mathbb{R}_{\geq a}$. By the already proved result for $C_{c}^{\infty}(\mathbb{R} ; H)$, we infer that $\operatorname{spt}\left(\partial_{0, \varrho}^{-\alpha} F_{\varrho}\left(u_{n}\right)-\partial_{0, \varrho}^{-\alpha} F_{\varrho}\left(v_{n}\right)\right) \subseteq \mathbb{R}_{\geq a}$ for all $n \in \mathbb{N}$. Thus, letting $n \rightarrow \infty$, we obtain $\operatorname{spt}\left(\partial_{0, \varrho}^{-\alpha} F_{\varrho}(u)-\partial_{0, \varrho}^{-\alpha} F_{\varrho}(v)\right) \subseteq \mathbb{R}_{\geq a}$, which shows the claim.

Finally, we prove that our solution is independent of the particular choice of the parameter $\varrho>v$ in Theorem 4.2. The precise statement is as follows.

Proposition 4.7 Let the assumptions of Theorem 4.2 be satisfied and $v$ be chosen according to Theorem 4.2. Let $\tilde{\mu}, \mu>v$ and $u_{\tilde{\mu}} \in H_{\tilde{\mu}}^{\beta}(\mathbb{R} ; H), u_{\mu} \in H_{\mu}^{\beta}(\mathbb{R} ; H)$ satisfying

$$
\partial_{0, \tilde{\mu}}^{\alpha} u_{\tilde{\mu}}=F_{\widetilde{\mu}}\left(u_{\tilde{\mu}}\right) \text { and } \partial_{0, \mu}^{\alpha} u_{\mu}=F_{\mu}\left(u_{\mu}\right) .
$$

Then $u_{\tilde{\mu}}=u_{\mu}$ as distributions in the sense of Proposition 3.4. 
Proof We note that it suffices to show $v_{\mu}:=\partial_{0, \mu}^{\beta} u_{\mu}=\partial_{0, \tilde{\mu}}^{\beta} u_{\tilde{\mu}}=: v_{\tilde{\mu}}$ as $L_{\text {loc }}^{1}(\mathbb{R} ; H)$ functions by Proposition 3.10. We consider the function

$$
\widetilde{F}: \operatorname{dom}(\widetilde{F}) \subseteq \bigcap_{\varrho \geq \varrho_{0}} H_{\varrho}^{0}(\mathbb{R} ; H) \rightarrow \bigcap_{\varrho \geq \varrho_{0}} H_{\varrho}^{\beta-\alpha}(\mathbb{R} ; H)
$$

given by

$$
\widetilde{F}(v):=F\left(\partial_{0, \varrho}^{-\beta} v\right) \quad(v \in \operatorname{dom}(\widetilde{F}))
$$

with maximal domain

$$
\operatorname{dom}(\widetilde{F})=\left\{w \in \bigcap_{\varrho \geq \varrho_{0}} H_{\varrho}^{0}(\mathbb{R} ; H) ; \forall \varrho \geq \varrho_{0}: \partial_{0, \varrho}^{-\beta} w \in \operatorname{dom}(F)\right\} .
$$

Note that the expression on the right hand side of (4.2) does not depend on the particular choice of $\varrho \geq \varrho_{0}$ by Proposition 3.10. Clearly, $\widetilde{F}$ is eventually $(0, \beta-\alpha)$-contracting (see also Remark 4.1(b)) and

$$
\widetilde{F}_{\varrho}=F_{\varrho}\left(\partial_{0, \varrho}^{-\beta}(\cdot)\right) \quad\left(\varrho \geq \varrho_{0}\right) .
$$

In particular,

$$
\partial_{0, \mu}^{\alpha-\beta} v_{\mu}=\partial_{0, \mu}^{\alpha} u_{\mu}=F_{\mu}\left(u_{\mu}\right)=\widetilde{F}_{\mu}\left(v_{\mu}\right)
$$

and analogously

$$
\partial_{0, \widetilde{\mu}}^{\alpha-\beta} v_{\widetilde{\mu}}=\widetilde{F}_{\widetilde{\mu}}\left(v_{\widetilde{\mu}}\right) .
$$

Let now $a \in \mathbb{R}$ and assume without loss of generality that $\mu<\widetilde{\mu}$. We note that $\operatorname{spt}\left(v_{\tilde{\mu}}-\right.$ $\left.\chi_{\mathbb{R}_{\leq} a} v_{\tilde{\mu}}\right) \subseteq \chi_{\mathbb{R}_{\geq a}}$. We obtain, applying Theorem 4.6, that

$$
\chi_{\mathbb{R}_{\leq a}} v_{\widetilde{\mu}}=\chi_{\mathbb{R}_{\leq a}} \partial_{0, \tilde{\mu}}^{\beta-\alpha} \widetilde{F}_{\widetilde{\mu}}\left(v_{\tilde{\mu}}\right)=\chi_{\mathbb{R}_{\leq a}} \partial_{0, \widetilde{\mu}}^{\beta-\alpha} \widetilde{F}_{\widetilde{\mu}}\left(\chi_{\mathbb{R}_{\leq a}} v_{\tilde{\mu}}\right) .
$$

Now, since $\chi_{\mathbb{R}_{\leq a}} v_{\widetilde{\mu}} \in L_{\mu}^{2}(\mathbb{R} ; H) \cap L_{\widetilde{\mu}}^{2}(\mathbb{R} ; H)$, we infer that

$$
\chi_{\mathbb{R}_{\leq a}} v_{\widetilde{\mu}}=\chi_{\mathbb{R}_{\leq a}} \partial_{0, \tilde{\mu}}^{\beta-\alpha} \widetilde{F}_{\widetilde{\mu}}\left(\chi_{\mathbb{R}_{\leq a}} v_{\tilde{\mu}}\right)=\chi_{\mathbb{R}_{\leq a}} \partial_{0, \mu}^{\beta-\alpha} \widetilde{F}_{\mu}\left(\chi_{\mathbb{R}_{\leq a}} v_{\tilde{\mu}}\right),
$$

i.e. $\chi_{\mathbb{R}_{\leq a}} v_{\tilde{\mu}}$ is a fixed point of $\chi_{\mathbb{R}_{\leq a}} \partial_{0, \mu}^{\beta-\alpha} \widetilde{F}_{\mu}$. However, since $\chi_{\mathbb{R}_{\leq a}} v_{\mu}$ is also a fixed point of this mapping, which is strictly contractive, we derive

$$
\chi_{\mathbb{R}_{\leq a}} v_{\widetilde{\mu}}=\chi_{\mathbb{R}_{\leq a}} v_{\mu}
$$

and since $a \in \mathbb{R}$ was arbitrary, the assertion follows.

\section{Riemann-Liouville and Caputo Differential Equations}

As it has been slightly touched in the introduction, there are two main concepts of fractional differentiation (or integration). In this section we shall start to identify both these notions as being part of the same solution theory developed in the previous sections. More precisely, equipped with the results from the previous sections we will consider the initial value problems for the Riemann-Liouville and for the Caputo derivative. In order to avoid subtleties as much as possible, we will consider the associated integral equations for both 
the Riemann-Liouville differential equation and the Caputo differential equation and reformulate these equivalently with the description of our realisation of the time-derivative and derive the well-posedness from our abstract solution theory.

To start off, we recall the Caputo differential equation. In [2,3], the author treated the following initial value problem of Caputo type for $\alpha \in] 0,1]$ :

$$
\begin{aligned}
D_{*}^{\alpha} y(t) & =f(t, y(t)) \quad(t>0) \\
y(0) & =y_{0},
\end{aligned}
$$

where a solution $y$ is continuous at zero and $y_{0} \in \mathbb{C}^{n}$ is a given initial value; $f: \mathbb{R}_{>0} \times \mathbb{C}^{n} \rightarrow$ $\mathbb{C}^{n}$ is continuous, satisfying

$$
\left|f\left(t, y_{1}\right)-f\left(t, y_{2}\right)\right| \leq c\left|y_{1}-y_{2}\right|
$$

for some $c \geq 0$ and all $y_{1}, y_{2} \in \mathbb{C}^{n}, t>0$. For definiteness, we shall also assume that

$$
(t \mapsto f(t, 0)) \in L_{\varrho_{0}}^{2}\left(\mathbb{R}_{>0} ; \mathbb{C}^{n}\right)
$$

for some $\varrho_{0} \in \mathbb{R}$. In order to circumvent discussions of how to interpret the initial condition, we shall rather put [2, Equation (6)] into the perspective of the present exposition. In fact, this equation reads in our notation and under the assumption $\alpha \in] 0,1]$

$$
y(t)=y_{0}+\frac{1}{\Gamma(\alpha)} \int_{0}^{t}(t-s)^{\alpha-1} f(s, y(s)) \mathrm{d} s \quad(t>0) .
$$

First of all, we remark that in contrast to the setting in the previous section, the differential equation just discussed 'lives' on $\mathbb{R}_{>0}$, only. To this end we put

$$
\tilde{f}: \mathbb{R} \times \mathbb{C}^{n} \rightarrow \mathbb{C}^{n}, \quad(t, y) \mapsto \chi_{\mathbb{R}_{>0}}(t) f(t, y),
$$

with the apparent meaning that $\tilde{f}$ vanishes for negative times $t$. We note that by (5.1) and (5.2) it follows that

$$
L_{\varrho}^{2}(\mathbb{R}) \ni y \mapsto(t \mapsto \tilde{f}(t, y(t))) \in L_{\varrho}^{2}(\mathbb{R})
$$

is a well-defined Lipschitz continuous mapping for all $\varrho \geq \varrho_{0}$. Obviously, (5.3) is equivalent to

$$
y(t)=y_{0} \chi_{\mathbb{R}_{>0}}(t)+\frac{1}{\Gamma(\alpha)} \int_{-\infty}^{t}(t-s)^{\alpha-1} \tilde{f}(s, y(s)) \mathrm{d} s \quad(t>0),
$$

which in turn can be (trivially) stated for all $t \in \mathbb{R}$. Next, we present the desired reformulation of equation (5.4).

Theorem 5.1 Let $\varrho>\max \left\{0, \varrho_{0}\right\}$. Assume that $y \in L_{\varrho}^{2}(\mathbb{R})$. Then the following statements are equivalent:

(i) $y(t)=y_{0} \chi_{\mathbb{R}_{>0}}(t)+\frac{1}{\Gamma(\alpha)} \int_{-\infty}^{t}(t-s)^{\alpha-1} \tilde{f}(s, y(s)) \mathrm{d}$ f for almost every $t \in \mathbb{R}$,

(ii) $y=\partial_{0, \varrho}^{-\alpha} \tilde{f}(\cdot, y(\cdot))+y_{0} \chi_{\mathbb{R}_{>0}}$,

(iii) $\partial_{0, \varrho}^{\alpha}\left(y-y_{0} \chi_{\mathbb{R}_{>0}}\right)=\widetilde{f}(\cdot, y(\cdot))$.

Proof The assertion follows trivially from Theorem 2.6.

Remark 5.2 (a) For a real-valued function $g: \mathbb{R}_{>0} \times \mathbb{R}^{n} \rightarrow \mathbb{R}^{n}$ we may consider the Caputo differential equation with $f: \mathbb{R}_{>0} \times \mathbb{C}^{n} \rightarrow \mathbb{C}^{n},(t, z) \mapsto g(t, \operatorname{Re}(z))$. 
(b) In particular, we have shown in Theorem 5.1 that the notions of so-called mild and strong solutions coincide.

Next we introduce Riemann-Liouville differential equations. Using the exposition in [12], we want to discuss the Riemann-Liouville fractional differential equation given by

$$
\begin{aligned}
\frac{d^{\alpha}}{d x^{\alpha}} y(x) & =f(x, y(x)), \\
\left.\frac{d^{\alpha-1}}{d x^{\alpha-1}} y(x)\right|_{x=0+} & =y_{0},
\end{aligned}
$$

where as before $f$ satisfies (5.1) and (5.2) and $y_{0} \in \mathbb{R}$, and $\left.\left.\alpha \in\right] 0,1\right]$. Again, not hinging on too much of an interpretation of this equation, we shall rather reformulate the equivalent integral equation related to this initial value problem. According to [12, Chapter 42] this initial value problem can be formulated as

$$
y(t)=y_{0} \frac{t^{\alpha-1}}{\Gamma(\alpha)}+\frac{1}{\Gamma(\alpha)} \int_{0}^{t}(t-s)^{\alpha-1} f(s, y(s)) \mathrm{d} s \quad(t>0) .
$$

We abbreviate $g_{\beta}(t):=\frac{1}{\Gamma(\beta+1)} t^{\beta} \chi_{\mathbb{R}_{>0}}(t)$ for $t, \beta \in \mathbb{R}$. For $\alpha>1 / 2$ we have $g_{\alpha-1} \in$ $L_{\varrho}^{2}(\mathbb{R} ; H)$. Let us assume that $\alpha>1 / 2$. Invoking the cut-off function $\chi_{\mathbb{R}_{>0}}$ and defining $\tilde{f}$ as before, we may provide a reformulation of the Riemann-Liouville equation on the space $L_{\varrho}^{2}(\mathbb{R} ; H)$ by

$$
y=g_{\alpha-1} y_{0}+\partial_{0, \varrho}^{-\alpha} f(\cdot, y(\cdot)), \quad y \in L_{\varrho}^{2}(\mathbb{R} ; H) .
$$

By a formal calculation and when applying Corollary 2.7, i.e. $\partial_{0, \varrho}^{-\alpha} \chi_{\mathbb{R}_{>0}} y_{0}=g_{\alpha} y_{0}$, we would obtain

$$
g_{\alpha-1} y_{0}=\partial_{0, \varrho} g_{\alpha} y_{0}=\partial_{0, \varrho} \partial_{0, \varrho}^{-\alpha} \chi_{\mathbb{R}_{>0}} y_{0}=\partial_{0, \varrho}^{-\alpha} \partial_{0, \varrho} \chi_{\mathbb{R}_{>0}} y_{0}=\partial_{0, \varrho}^{-\alpha} y_{0} \delta_{0},
$$

where $\partial_{0, \varrho} \chi_{\mathbb{R}_{>0}} y_{0}$ is, when understood distributionally, the delta function $y_{0} \delta_{0}$ and we could reformulate the Riemann-Liouville equation by

$$
\partial_{0, \varrho}^{\alpha} y=y_{0} \delta_{0}+\tilde{f}(\cdot, y(\cdot)) .
$$

In order to apply our solution theory, we need the following result.

Proposition 5.3 Let $\varrho_{0}>0, n \in \mathbb{N}, y_{0} \in \mathbb{R}^{n}, f: \mathbb{R}_{>0} \times \mathbb{C}^{n} \rightarrow \mathbb{C}^{n}$ continuous. Assume there exists $c \geq 0$ such that for all $y_{1}, y_{2} \in \mathbb{R}^{n}, t>0$ we have

$$
\left|f\left(t, y_{1}\right)-f\left(t, y_{2}\right)\right| \leq c\left|y_{1}-y_{2}\right| \text {. }
$$

Moreover, we assume that

$$
(t \mapsto f(t, 0)) \in L_{\varrho_{0}}^{2}\left(\mathbb{R}_{>0} ; \mathbb{C}^{n}\right) .
$$

Define $\tilde{f}: \mathbb{R} \times \mathbb{C}^{n} \rightarrow \mathbb{C}^{n}$ by

$$
\tilde{f}(t, y):= \begin{cases}f(t, y) & \text { if } t>0, \\ 0 & \text { else. }\end{cases}
$$

Then the mapping $F: C_{c}^{\infty}\left(\mathbb{R} ; \mathbb{C}^{n}\right) \rightarrow C\left(\mathbb{R} ; \mathbb{C}^{n}\right)$ given by

$$
F(\varphi)(t):=\tilde{f}\left(t, \varphi(t)+y_{0}\right) \quad\left(\varphi \in C_{c}^{\infty}\left(\mathbb{R} ; \mathbb{C}^{n}\right), t \in \mathbb{R}\right),
$$

is eventually $(0,0)$-Lipschitz continuous. 
Proof Let $\varrho \geq \varrho_{0}$. In order to prove that $F$ attains values in $L_{\varrho}^{2}\left(\mathbb{R} ; \mathbb{C}^{n}\right)$, we shall show $F(0) \in L_{\varrho}^{2}\left(\mathbb{R} ; \mathbb{C}^{n}\right)$ first. For this we compute

$$
\begin{aligned}
\int_{\mathbb{R}}|F(0)(t)|^{2} \mathrm{e}^{-2 \varrho t} \mathrm{~d} t & =\int_{\mathbb{R}_{>0}}\left|f\left(t, y_{0}\right)\right|^{2} \mathrm{e}^{-2 \varrho t} \mathrm{~d} t \\
& \leq 2\left(\int_{\mathbb{R}_{>0}}\left|f\left(t, y_{0}\right)-f(t, 0)\right|^{2} \mathrm{e}^{-2 \varrho t} \mathrm{~d} t+\int_{\mathbb{R}_{>0}}|f(t, 0)|^{2} \mathrm{e}^{-2 \varrho t} \mathrm{~d} t\right) \\
& \leq 2\left(c^{2}\left|y_{0}\right|^{2} \frac{1}{2 \varrho}+|f(\cdot, 0)|_{L_{\varrho 0}^{2}\left(\mathbb{R}_{>0} ; \mathbb{C}^{n}\right)}^{2}\right)<\infty .
\end{aligned}
$$

Here we used that $L_{\varrho}^{2}\left(\mathbb{R}_{>0} ; H\right) \hookrightarrow L_{\varrho 0}^{2}\left(\mathbb{R}_{>0} ; H\right)$ as contraction. Next, let $\varphi, \psi \in$ $C_{c}^{\infty}\left(\mathbb{R} ; \mathbb{R}^{n}\right)$. Then we obtain

$$
\begin{aligned}
\int_{\mathbb{R}}|F(\varphi)(t)-F(\psi)(t)|^{2} \mathrm{e}^{-2 \varrho t} \mathrm{~d} t & =\int_{\mathbb{R}}\left|\tilde{f}\left(t, \varphi(t)+y_{0}\right)-\tilde{f}\left(t, \psi(t)+y_{0}\right)\right|^{2} \mathrm{e}^{-2 \varrho t} \mathrm{~d} t \\
& =\int_{\mathbb{R}_{>0}}\left|f\left(t, \varphi(t)+y_{0}\right)-f\left(t, \psi(t)+y_{0}\right)\right|^{2} \mathrm{e}^{-2 \varrho t} \mathrm{~d} t \\
& \leq \int_{\mathbb{R}_{>0}} c^{2}(|\varphi(t)-\psi(t)|)^{2} \mathrm{e}^{-2 \varrho t} \mathrm{~d} t \leq c^{2}\|\varphi-\psi\|_{L_{\varrho}^{2} .}^{2}
\end{aligned}
$$

Since $F(0) \in L_{\varrho}^{2}\left(\mathbb{R} ; \mathbb{C}^{n}\right)$, the shown estimate yields $F(\varphi) \in L_{\varrho}^{2}\left(\mathbb{R} ; \mathbb{C}^{n}\right)$ for each $\varphi \in$ $C_{c}^{\infty}\left(\mathbb{R} ; \mathbb{C}^{n}\right)$ as well as the eventual $(0,0)$-Lipschitz continuity of $F$.

The next result is concerned with the well-posedness for Caputo fractional differential equations. We shall use the characterization of the Caputo differential equation outlined in Theorem 5.1.

Theorem 5.4 Let $y_{0} \in \mathbb{C}^{n}$. Then there is $\varrho_{1}>0$ such that for all $\varrho \geq \varrho_{1}$ there exists a unique $y \in L_{\varrho}^{2}\left(\mathbb{R} ; \mathbb{C}^{n}\right)$ with $y-y_{0} \chi_{\mathbb{R}_{>0}} \in H_{\varrho}^{\alpha}\left(\mathbb{R} ; \mathbb{C}^{n}\right)$ satisfying

$$
\partial_{0, \varrho}^{\alpha}\left(y-y_{0} \chi_{\mathbb{R}_{>0}}\right)=\tilde{f}(\cdot, y(\cdot)) .
$$

Moreover, spt $y \subseteq \mathbb{R}_{\geq 0}$.

Proof With $F$ as defined in Proposition 5.3, we may apply Corollary 4.3 with $\beta=\gamma=0$ to obtain unique existence of $z \in H_{\varrho}^{\alpha}\left(\mathbb{R} ; \mathbb{C}^{n}\right)$ such that

$$
\partial_{0, \varrho}^{\alpha} z=F_{\varrho}(z) \text {. }
$$

Setting $y:=z+y_{0} \chi_{\mathbb{R}_{>0}}$, we obtain in turn unique existence of a solution of the desired equation. Since spt $F_{\varrho}(z) \subseteq \mathbb{R}_{\geq 0}$, we obtain with Proposition 4.5 that spt $z=\operatorname{spt} \partial_{0, \varrho}^{-\alpha} F_{\varrho}(z) \subseteq \mathbb{R}_{\geq 0}$. Thus, spt $y \subseteq \mathbb{R}_{\geq 0}$.

We remark here that the condition spt $y \subseteq \mathbb{R}_{\geq 0}$ together with $y-y_{0} \chi_{\mathbb{R}_{>0}} \in H_{\varrho}^{\alpha}\left(\mathbb{R} ; \mathbb{C}^{n}\right)$ describes, how the initial value $y_{0}$ is attained. Indeed, if $\alpha$ is large enough (e.g. $\alpha>1 / 2$ ) so that $H_{\varrho}^{\alpha}\left(\mathbb{R} ; \mathbb{C}^{n}\right)$ is a subset of functions for which the limit at 0 exists, then the mentioned conditions imply

$$
0=\left(y-y_{0} \chi_{\mathbb{R}_{>0}}\right)(0-)=\left(y-y_{0} \chi_{\mathbb{R}_{>0}}\right)(0+)=y(0+)-y_{0},
$$

that is, the initial value is attained. 
We conclude this section by having a look at the case of the Riemann-Liouville fractional differential equation (5.5). To this end, we note that $\chi_{\mathbb{R}_{>0}} y_{0} \in H_{\varrho}^{0}(\mathbb{R} ; H)$ for $\varrho>0$ and by Example 3.6 we have

$$
\partial_{0, \varrho} \chi_{\mathbb{R}_{>0}} y_{0}=\delta_{0} y_{0} \in H_{\varrho}^{-1}(\mathbb{R} ; H) .
$$

We also recall the notation $g_{\beta}(t):=\frac{1}{\Gamma(\beta+1)} t^{\beta} \chi_{\mathbb{R}_{>0}}$ for $\beta, t \in \mathbb{R}$.

Proposition 5.5 Let $y_{0} \in \mathbb{C}^{n}$. Assume that $C_{c}^{\infty}(\mathbb{R} ; H) \ni \varphi \mapsto \tilde{f}(\cdot, \varphi(\cdot))$ is eventually $(\alpha-1, \alpha-1)$-Lipschitz continuous and denote with $H_{\varrho}^{\alpha-1}(\mathbb{R} ; H) \ni y \mapsto \widetilde{f}_{\varrho}(\cdot, y(\cdot))$ its Lipschitz-continuous extension for some $\varrho>0$. There is $\varrho_{1}>0$ such that for $\varrho \geq \varrho_{1}$ we have a unqiue solution $y \in H_{\varrho}^{\alpha-1}(\mathbb{R} ; H)$ of the equation

$$
\partial_{0, \varrho}^{\alpha} y=y_{0} \delta_{0}+\widetilde{f}_{\varrho}(\cdot, y(\cdot)), \quad y \in H_{\varrho}^{\alpha-1}(\mathbb{R} ; H),
$$

with $\partial_{0, \varrho}^{\alpha-1} y-y_{0} \chi_{\mathbb{R}_{>0}} \in H_{\varrho}^{0}(\mathbb{R} ; H)$ and $\operatorname{spt}(y) \subseteq \mathbb{R}_{\geq 0}$.

Proof The mapping $G$ defined by

$$
G(\varphi)(t):=\tilde{f}\left(t, \partial_{0, \varrho}^{1-\alpha} \varphi(t)+g_{\alpha-1}(t) y_{0}\right), \quad \varphi \in C_{c}^{\infty}(\mathbb{R} ; H), t \in \mathbb{R},
$$

is eventually $(0, \alpha-1)$-Lipschitz continuous. Indeed, this fact follows from $g_{\alpha-1} y_{0} \in$ $H_{\varrho}^{\alpha-1}(\mathbb{R} ; H)$ and the unitarity of $\partial_{0, \varrho}^{\alpha-1}: H_{\varrho}^{\alpha-1}(\mathbb{R} ; H) \rightarrow H_{\varrho}^{0}(\mathbb{R} ; H)$. Let $\varrho_{1}>0$ be such that $\widetilde{f}_{\varrho}$ and therefore $G_{\varrho}$ exist for $\varrho \geq \varrho_{1}$. Let $\varrho \geq \varrho_{1}$. The Riemann-Liouville equation is equivalent to

$$
\partial_{0, \varrho}^{\alpha-1} y-\chi_{\mathbb{R}_{>0}} y_{0}=\partial_{0, \varrho}^{-1} \widetilde{f}_{\varrho}(\cdot, y(\cdot)), \quad y \in H_{\varrho}^{\alpha}(\mathbb{R} ; H) .
$$

With the transformation $z=\partial_{0, \varrho}^{\alpha-1} y-\chi_{\mathbb{R}_{>0}} y_{0}$ and using $\partial_{0, \varrho}^{1-\alpha} \chi_{\mathbb{R}_{>0}} y_{0}=\partial_{0, \varrho} g_{\alpha} y_{0}=g_{\alpha-1} y_{0}$ (cf. Corollary 2.7) this equation is equivalent to

$$
\partial_{0, \varrho} z=G_{\varrho}(z), \quad z \in H_{\varrho}^{0}(\mathbb{R} ; H) .
$$

By Corollary 4.3 (with $\gamma=\alpha-1$ ) we find a unique solution $z \in H_{\varrho}^{0}(\mathbb{R} ; H)$. We have $\operatorname{spt}\left(\partial_{0, \varrho}^{-1} G_{\varrho}(\cdot)\right) \subseteq \mathbb{R}_{\geq 0}$. By Proposition $4.5 \mathrm{spt} z \subseteq \mathbb{R}_{\geq 0}$. Hence we have a unqiue solution $y=\partial_{0, \varrho}^{1-\alpha}\left(z-\chi_{\mathbb{R}_{>0}} y_{0}\right) \in H_{\varrho}^{\alpha-1}(\mathbb{R} ; H)$ of the Riemann-Liouville equation with spt $y \subseteq \mathbb{R}_{\geq 0}$ and $\partial_{0, \varrho}^{\alpha-1} y-\chi_{\mathbb{R}_{>0}} y_{0}=z \in H_{\varrho}^{0}(\mathbb{R} ; H)$.

Remark 5.6 The space $H_{\varrho}^{0}(\mathbb{R} ; H)$ is continuously embedded into $H_{\varrho}^{\alpha-1}(\mathbb{R} ; H)$. Thus, the assumption that $C_{c}^{\infty}(\mathbb{R} ; H) \ni \varphi \mapsto \tilde{f}(\cdot, \varphi(\cdot))$ is eventually $(\alpha-1, \alpha-1)$-Lipschitz continuous, can be replaced by the stronger assumption that $C_{c}^{\infty}(\mathbb{R} ; H) \ni \varphi \mapsto \widetilde{f}(\cdot, \varphi(\cdot))$ is eventually $(\alpha-1,0)$-Lipschitz continuous, which might be easier to compute.

Funding Open Access funding enabled and organized by Projekt DEAL.

Open Access This article is licensed under a Creative Commons Attribution 4.0 International License, which permits use, sharing, adaptation, distribution and reproduction in any medium or format, as long as you give appropriate credit to the original author(s) and the source, provide a link to the Creative Commons licence, and indicate if changes were made. The images or other third party material in this article are included in the article's Creative Commons licence, unless indicated otherwise in a credit line to the material. If material is not included in the article's Creative Commons licence and your intended use is not permitted by statutory regulation or exceeds the permitted use, you will need to obtain permission directly from the copyright holder. To view a copy of this licence, visit http://creativecommons.org/licenses/by/4.0/. 


\section{References}

1. Akhiezer, N.I., Glazman, I.M.: Theory of linear operators in Hilbert space. Vol. I, II. Transl. from the 3rd Russian ed. by E. R. Dawson, W. N. Everitt. Monographs and Studies in Mathematics, 9, 10. Publ. in association with Scottish Academic Press, Edinburgh. Boston-London-Melbourne: Pitman Advanced Publishing Program. XXXII (1981)

2. Diethelm, K.: Efficient solution of multi-term fractional differential equations using $\mathrm{P}(\mathrm{EC})^{m} \mathrm{E}$ methods. Computing 71(4), 305-319 (2003)

3. Diethelm, K.: Fractional Differential Equations. Springer, Berlin (2010)

4. Gorenflo, R., Luchko, Y., Yamamoto, M.: Time-fractional diffusion equation in the fractional Sobolev spaces. Fract. Calc. Appl. Anal. 18(3), 799-820 (2015)

5. Kalauch, A., Picard, R., Siegmund, S., Trostorff, S., Waurick, M.: A Hilbert space perspective on ordinary differential equations with memory term. J. Dyn. Differ. Equ. 26(2), 369-399 (2014)

6. Morgenstern, D.: Beiträge zur nichtlinearen Funktionalanalysis. PhD thesis, TU Berlin (1952)

7. Ortigueira, M.D.: Fractional Calculus for Scientists and Engineers. Springer, Dordrecht (2011)

8. Paley, R., Wiener, N.: Fourier transforms in the complex domain. Colloq. Publ. Amer. Math. Soc. (1934)

9. Picard, R.: Hilbert Space Approach to Some Classical Transforms. Pitman Research Notes in Mathematics Series. 196. Harlow and New York: Longman Scientific \& Technical and Wiley (1989)

10. Picard, R., Trostorff, S., Waurick, M.: On evolutionary equations with material laws containing fractional integrals. Math. Methods Appl. Sci. 38(15), 3141-3154 (2015)

11. Rudin, W.: Real and Complex Analysis, 3rd edn. McGraw-Hill, New York, NY (1987)

12. Samko, S.G., Kilbas, A.A., Marichev, O.I.: Fractional Integrals and Derivatives. Gordon and Breach Science Publishers, Yverdon (1993). Theory and applications, Edited and with a foreword by S. M. Nikolski, Translated from the 1987 Russian original, Revised by the authors

13. Zacher, R.: Weak solutions of abstract evolutionary integro-differential equations in Hilbert spaces. Funkcial. Ekvac. 52(1), 1-18 (2009)

Publisher's Note Springer Nature remains neutral with regard to jurisdictional claims in published maps and institutional affiliations. 